CERN - TH/95-295

hep-th/9512125

December 1995

\title{
NEGATIVE SCREENINGS IN LIOUVILLE THEORY
}

\author{
Jens SCHNITTGER
}

CERN-TH, 1211 Geneva 23, Switzerland.

\begin{abstract}
We demonstrate how negative powers of screenings arise as a nonperturbative effect within the operator approach to Liouville theory. This explains the origin of the corresponding poles in the exact Liouville three point function proposed by Dorn/Otto and (Zamolodchikov) ${ }^{2}$ (DOZZ) and leads to a consistent extension of the operator approach to arbitrary integer numbers of screenings of both types. The general Liouville three point function in this setting is computed without any analytic continuation procedure, and found to support the DOZZ conjecture. We point out the importance of the concept of free field expansions with adjustable monodromies - recently advocated by Petersen, Rasmussen and $\mathrm{Yu}$ - in the present context, and show that it provides a unifying interpretation for two types of previously constructed local observables.
\end{abstract}




\section{Introduction and Summary}

In spite of remarkable progress in the understanding of quantum Liouville theory, the computation of correlation functions has remained a thorny issue. Nonrigorous analytic continuation techniques [1] [2] [3] [4] have provided results for genus zero and one amplitudes of minimal matter coupled to gravity which agree with those obtained from the matrix models. The extension of these techniques led to an expression for the general Liouville three point functions $\left\langle e^{\alpha_{1} \Phi} e^{\alpha_{2} \Phi} e^{\alpha_{3} \Phi}\right\rangle$ at arbitrary values of the central charge [5], which recently was rediscovered in [6]. However, a rigorous derivation of these results is still missing, and the analytic continuation method by its very nature prevents us from obtaining a true insight into the dynamical mechanism which leads to the complicated form of the Liouville correlation functions. The basic difficulty one encounters is that though naively, a Coulomb gas picture for the theory can be obtained by expanding in powers of the interaction term - which formally is a screening operator involving the semiclassical screening charge $\alpha_{-}-$, general Liouville correlators actually require the insertion of noninteger powers of $\alpha_{-}$screenings \&. The operator approach to Liouville theory, developed in a long series of papers [7], provides a well-defined framework within which it should be possible to address this problem. In [\$], general Liouville exponentials were constructed as infinite series in Coulombgas-like vertex operators, while no attempt was made yet to determine their correlation functions. In the present paper, we intend to make a first step towards a first principle derivation of Liouville correlators (within the elliptic sector usually considered, that is, for "microscopic" operators [9] 31]), by considering an important special case. We will analyze the three point function in the situation where the total screening charge $\alpha_{s}=2 \alpha_{0}-\left(\alpha_{1}+\alpha_{2}+\alpha_{3}\right)$ is given by a linear combination with integer coefficients $\alpha_{s}=s_{+} \alpha_{+}+s_{-} \alpha_{-}$ of the two elementary screening charges $\alpha_{+}$and $\alpha_{-}$, and the exponent $-2 \alpha$ of at least one of the Liouville exponentials $e^{\alpha_{i} \Phi}$ fulfills the same condition. (The degenerate sector of Liouville theory, studied extensively in the older works [1] [14, is recovered when these integers are positive). For irrational central charge where $\alpha_{+}$and $\alpha_{-}$are incommensurate, these values lie dense in the continuum. The reason for the restriction to integer screening numbers

\footnotetext{
${ }^{1}$ Another way of saying this is that the dependence of correlators on the cosmological constant is in general nonanalytic.
} 
of both types can be understood within the concept of multivalued free field expansions with arbitrary monodromy recently introduced in ref. [17] within the context of correlators for $S L(2)$ - Kac-Moody based theories. It provides, at the same time, a unifying interpretation for two apparently different kinds of local observables constructed in ref. [8]. The first type involves only the semiclassical screening charge $\alpha_{-}$and is continously connected to the classical Liouville exponentials by a naive classical limit. On the other hand, the DOZZ three point function is completely symmetric in $\alpha_{+}$and $\alpha_{-}$, just like the second kind of local observables of ref. [8], whose interpretation had remained unclear. The explanation we will propose is that the two types of observables are nothing but expansions with different monodromies of the same Liouville exponentials, to be chosen according to whether the matrix element considered involves $\alpha_{+}$-screenings or not.

In our analysis we will distinguish two basic cases: If (at least) one of the four conditions $s_{ \pm} \geq 0, s_{ \pm}<0, s_{ \pm} \leq-p_{ \pm}$, or $s_{ \pm}>-p_{ \pm}$is fulfilled, where $p_{ \pm}$characterize the integer-valued exponent $2 \alpha=p_{+} \alpha_{+}+p_{-} \alpha_{-}$, then the DOZZ three point function has a pole; otherwise it is regular as a function of the screening charge $\alpha_{s}$ 2. Only the poles at positive $s_{-}$and $s_{+}=0$ are expected within the naive perturbative picture as resulting from a charge conservation $\delta$ function for the zero mode integration, while the occurrence of poles at $s_{-}<0$ and at $s_{+} \neq 0$ is surprising. The concept of variable monodromy expansions, together with locality and the complete symmetry of the conformal structure under exchange of $\alpha_{+}$and $\alpha_{-}$, provides some explanation for the latter, but not for the appearance of poles at negative screenings. However, we will derive that the series expansions of ref. [8] for the Liouville exponentials, which superficially involve only positive powers of screenings, actually simulate the effect of negative screenings when evaluated carefully, due to the nontrivial convergence properties of the infinite series. Indeed, using a coherent state basis (eigenstates of the screening operators) one finds that the zero mode integration can be transformed into a contour integral on the unit circle over the eigenvalue of the screening operator. The residue at the origin corresponds to a naive ("perturbative") term by term zero mode integration of the series representing the Liouville exponential, while the other residues represent nonperturbative effects which break the charge conservation rules of the naive perturbative picture. This interesting

\footnotetext{
${ }^{2}$ (in the generic case when only one of the exponents $2 \alpha_{i}$ is integer-valued)
} 
mechanism explains the appearance of poles in the DOZZ three point function at negative values of the screening numbers, the singularity coming simply from the infinite zero mode volume. In the present analysis, where we restrict effectively to a discrete spectrum, the zero mode volume is treated as finite, and we obtain the residues of the DOZZ result at the poles. The inclusion of negative powers of screenings leads to an extension of the operator approach as elaborated in recent works [8] 13] 12] [14. It is an important virtue of the Gervais-Neveu quantization scheme that negative powers of screenings can be formulated without the need for analytic continuation. Indeed, positive and negative screening powers are related to the two canonically equivalent fields introduced long ago [20]. All observables must be completely symmetric under the exchange of the two free fields, which can be associated with a Weyl reflection with respect to the underlying $U_{q}(s l(2))$ symmetry of the theory, and this serves as an important guiding principle for the nonperturbative definition of the Liouville exponentials. We will show that the three point function is invariant under this reflection, so that highest weight states which it relates can be consistently identified, as expected from the two to one nature of the Bäcklund transformation relating the Liouville to the free field. The resulting theory lives exactly on the poles of the DOZZ three point function and fulfills the crossing symmetry/locality conditions. On the other hand, in the cases where the DOZZ three point function is finite, the sum of the residues vanishes; this is of course again a consequence of the different treatment of the zero mode volume. However, we will point out briefly that in fact it is possible to obtain the DOZZ result at these points as well by a formal renormalization of the expansion coefficients of the Liouville exponentials. The resulting "renormalized exponentials", which are again local, turn out to reproduce as a special case the dressing operators proposed in ref. [7] for the coupling of minimal matter to gravity.

\section{Liouville exponentials}

We start by briefly recalling the results of ref. [8] that will be needed here. Liouville fields $e^{-J \alpha_{-} \Phi}$ with arbitrary spin $J$ and conformal weight $\Delta_{J}=$

$-J-\frac{h}{\pi} J(J+1)$ can be written as an infinite series in Coulomb gas vertex 
operators:

$$
\exp \left[-J \alpha_{-} \Phi(\sigma, \tau)\right]=\sum_{n=0}^{\infty} \frac{T(\varpi)}{T(\varpi+2 n-2 J)} a_{n}^{(J)}(\varpi) V_{-J}^{(J)}(u) \bar{V}_{-J}^{(J)}(v) S(u)^{n} \bar{S}(v)^{n}
$$

Here $\varpi$ denotes the (rescaled) free field zero mode which is real in the elliptic sector considered here, $u=\tau+\sigma$ and $v=\tau-\sigma$ are light cone variables formed from the usual cylinder coordinates $\tau$ and $\sigma$, and the coefficient $a_{n}^{(J)}(\varpi)$ is given by

$$
a_{n}^{(J)}(\varpi)=\left(\frac{\Gamma(1-h / \pi)}{2 \pi}\right)^{2 n} \frac{\lfloor-2 J\rfloor_{n}}{\lfloor n\rfloor !\lfloor\varpi+1\rfloor_{n}\lfloor\varpi-2 J+n\rfloor_{n}}
$$

with

$$
\lfloor x\rfloor:=\frac{\sin (h x)}{\sin (h)}, \quad\lfloor a\rfloor_{n}:=\prod_{k=0}^{n-1}\lfloor a+k\rfloor
$$

The effective Planck constant $h$ is related to the central charge $C$ by

$$
h=\frac{\pi}{12}(C-13-\sqrt{(C-25)(C-1)})
$$

As usual, $\alpha_{-}$denotes the semiclassical screening charge, given in terms of $h$ by

$$
\alpha_{-}=\sqrt{\frac{2 h}{\pi}}
$$

Furthermore, the Coulomb gas vertex operators are defined as

$$
V_{-J}^{(J)}(u)=: e^{2 J \sqrt{h / 2 \pi} \vartheta_{1}}:
$$

where $\vartheta_{1}$ is a chiral free field obeying

$$
\left[\vartheta_{1}^{\prime}\left(u_{1}\right), \vartheta_{1}^{\prime}\left(u_{2}\right)\right]=2 \pi i \delta^{\prime}\left(u_{1}-u_{2}\right)
$$

(the meaning of the index 1 will become clear later on) and

$$
S(u)=e^{2 i h(\varpi+1)} \int_{0}^{u} d u^{\prime} V_{1}^{(-1)}\left(u^{\prime}\right)+\int_{u}^{2 \pi} d u^{\prime} V_{1}^{(-1)}\left(u^{\prime}\right) .
$$

$S(u)$ is a dimension zero primary field, called screening operator in the sequel. Similar formulae hold for the right-moving vertex operators. We note that 
powers of $S$ are well-defined without renormalization' and the same is true for the product $V_{-J}^{(J)}(u) S^{n}(u)$. The Coulomb gas operators shift the zero mode by

$$
V_{-J}^{(J)} \varpi=(\varpi-2 J) V_{-J}^{(J)}
$$

and

$$
S \varpi=(\varpi+2) S,
$$

Finally, let us turn to the factor $\frac{T(\varpi)}{T(\varpi+2 n-2 J)}$. In ref. 19 it was pointed out that the locality conditions which determine the coefficients $a_{n}^{(J)}(\varpi)$ possess an obvious invariance generated by the similarity transformations

$$
\exp \left[-J \alpha_{-} \Phi\right] \rightarrow T(\varpi) \exp \left[-J \alpha_{-} \Phi\right] T^{-1}(\varpi)
$$

with arbitrary (smooth) functions $T(\varpi)$, which, using Eqs. (8), lead to the first factor in Eq.(1). However, $T(\varpi)$ is not truly arbitrary but strongly constrained by imposing invariance under the discrete remnant of the underlying $S L(2)$-symmetry of the theory -see below. For reasons discussed in section 4, the expansion Eq. (1) should be used only for matrix elements of the type $\left\langle f_{\varpi_{f}}^{\prime}\left|\exp \left[-J \alpha_{-} \Phi\right]\right| f_{\varpi_{i}}\right\rangle, f_{\varpi}$ denoting an arbitrary state in the Fock module over the ground state $|\varpi\rangle$, with integer values of

$$
s:=J-\frac{1}{2}\left(\varpi_{f}-\varpi_{i}\right) \in \mathbf{Z}
$$

Eqs. (8) tell us that $s$ is the number of screening charges $\left(s \alpha_{-}=\alpha_{s}\right)$ needed to connect bra and ket in the above matrix element. Naively these matrix elements are nonzero only for nonnegative $s$ according to Eq. (1), but actually we will see that the formal infinite series in Eq. (1) effectively contains also negative screenings. On the other hand, if we consider the more general matrix elements with

$$
s \in \mathbf{Z}+\mathbf{Z} \frac{\pi}{h}
$$

with $\pi / h=\alpha_{+} / \alpha_{-}$equalling the ratio of the two screening charges, then we should use the expansion

$$
\exp \left[-J \alpha_{-} \Phi\right]=\sum_{n, \widehat{n}=0}^{\infty} \frac{T(\varpi)}{T\left(\varpi+2 m_{n, \widehat{n}}\right)} a_{n, \widehat{n}}^{(J)}(\varpi) V_{-J}^{(J)} S^{n} \widehat{S}^{\widehat{n}} \bar{V}_{-J}^{(J)} \bar{S}^{n} \widehat{\bar{S}}^{\widehat{n}}
$$

\footnotetext{
${ }^{3}$ (Up to a certain maximal power depending on $h$, but the Coulomb gas operators retain a well-defined meaning for any power by analytic continuation -see [8]).
} 
where $m_{n, \widehat{n}}:=-J+n+\widehat{n} \pi / h$ and

$$
\begin{gathered}
a_{n, \widehat{n}}^{(J)}(\varpi)=a_{n}^{(J)}(\varpi) \widehat{a}_{\widehat{n}}^{(\widehat{J})}(\widehat{\varpi}) \equiv \\
\left(\frac{\Gamma(1-h / \pi)}{2 \pi}\right)^{2 n} \frac{\lfloor-2 J\rfloor_{n}}{\lfloor n\rfloor !\lfloor\varpi+1\rfloor_{n}\lfloor\varpi-2 J+n\rfloor_{n}} \\
\times\left(\frac{\Gamma(1-\widehat{h} / \pi)}{2 \pi}\right)^{2 \widehat{n}} \frac{\hat{\lfloor}-2 \widehat{J}\rfloor_{\widehat{n}}}{\hat{\lfloor} \widehat{n} \hat{\rfloor} ! \hat{\lfloor} \widehat{\varpi}+1 \hat{\rfloor} \hat{\varpi}-2 \widehat{J}+\widehat{n} \hat{\rfloor}}
\end{gathered}
$$

Here we have put $\widehat{h}:=\frac{\pi^{2}}{h}, \hat{\lfloor} x \hat{\rfloor}:=\frac{\sin (\widehat{h} x)}{\sin (\widehat{h})}, \widehat{J}:=J \frac{h}{\pi}$ and $\widehat{\varpi}:=\varpi \frac{h}{\pi}$. Likewise, $\widehat{S}$ is given by Eq. (7) with $h$ replaced by $\widehat{h}$ everywhere. Eqs. (12), (13) are manifestly symmetric in $\alpha_{+}, \alpha_{-}$(or $h, \widehat{h}$ ) and so the preference of $\alpha_{-}$over $\alpha_{+}$on the left-hand side of Eq. (12) is purely notational. In using, different from ref. [8], the same designation for the local observables of Eq. (1) and Eq. (12), we anticipate the result of the discussion in section 1 which leads to their identification. We note that here and below, we consider only the case of zero winding number, that is, of equal values of left and right zero mode [8]. For later convenience, we introduce another, equivalent way of writing Eqs. (11) or (12] [8]:

$$
\exp \left[-J \alpha_{-} \Phi\right]=\sum_{\underline{n}=0}^{\infty} \frac{T(\varpi)}{T(\varpi+2 m)} g_{J, x+m}^{x} \bar{g}_{J, x+m}^{x} V_{m}^{(J)} \bar{V}_{m}^{(J)}
$$

where $m:=m_{n, \widehat{n}}$ with $\widehat{n}=0$ in the case of Eq. (1), and similarly $\underline{n}=$ $n, \widehat{n}$ or $\underline{n}=n$. Furthermore, $V_{m}^{(J)}$ denote normalized vertex operators with $\left\langle\varpi\left|V_{m}^{(J)}\right| \varpi+2 m\right\rangle=1$, related to the Coulomb gas vertex operators by

$$
V_{-J}^{(J)} S^{n} \widehat{S}^{\widehat{n}}=I_{m}^{(J)}(\varpi) V_{m}^{(J)}
$$

where the normalization factors $I_{m}^{(J)}(\varpi)$ are given in the appendix. The coefficients $g_{J, x+m}^{x}$ with $x:=\frac{1}{2}\left(\varpi-\varpi_{0}\right) \equiv \frac{1}{2}(\varpi-1-\pi / h)$ appear as coupling constants in the fusion and braiding algebra of the chiral vertex operators [14], [8]. Comparing Eq. (14) with Eq. (11) or Eq. (12) we have

$$
g_{J, x+m}^{x} \bar{g}_{J, x+m}^{x}=a_{\underline{n}}^{(J)}(\varpi)\left|I_{m}^{(J)}(\varpi)\right|^{2} .
$$




\section{Nonperturbative evaluation of matrix elements}

Due to conformal invariance [24], in order to define the operators Eq. (14) it is in principle sufficient to determine the ground state matrix element

$$
\left\langle\varpi_{f}\left|\exp \left[-J \alpha_{-} \Phi\right](\tau=\sigma=0)\right| \varpi_{i}\right\rangle
$$

with the screening number $s$ subject to Eq. (11) but otherwise arbitrary parameters. This matrix element is closely connected to the three point function via the formal relations

$$
\begin{gathered}
\left|\varpi_{i}\right\rangle=F_{i} \lim _{\tau \rightarrow-\infty}(z \bar{z})^{-\Delta_{J}}\left(\exp \left[-J_{i} \Phi(-i \tau, \sigma)\right]\right)_{\underline{n}=0}\left|\varpi_{0}\right\rangle \\
\left\langle\varpi_{f}\right|=F_{f} \lim _{\tau \rightarrow+\infty}(z \bar{z})^{\Delta_{J}}\left\langle-\varpi_{0}\right|\left(\exp \left[-J_{f} \Phi(-i \tau, \sigma)\right]\right)_{\underline{n}=0}
\end{gathered}
$$

Here, the subscript on the Liouville exponentials denotes the $n=\widehat{n}=0$ contribution in Eq. (14),

$$
z:=e^{\tau+i \sigma}, \quad \varpi_{0}:=1+\pi / h, \quad J_{f}=-\frac{1}{2}\left(\varpi_{0}+\varpi_{f}\right), \quad J_{i}=\frac{1}{2}\left(\varpi_{i}-\varpi_{0}\right)
$$

and $F_{i}, F_{f}$ are normalization factors which will be determined later. The prescription Eq. (18) relates operators to highest weight states of the same conformal dimension, as usual. However, in Liouville theory the operator state correspondence is actually a subtle issue [18] [9], and this is related to the problems with defining an $S L(2)$ - invariant vacuum in the present framework [21. . Therefore, at present Eq. (18) should be viewed as a formal prescription. In the degenerate case studied in the older works [24] [7], where the dimensions of the Liouville fields fall into Kac's table and the sums in Eq. (14) truncate, it is unnecessary to select the $\underline{n}=0$ contributions as all other ones are automatically suppressed in the limit $z \rightarrow 0$; however this is not the case in the present extended framework where divergent terms would appear.

In this section we will concentrate on the case where only $\alpha_{-}$screenings are present, i.e. $\widehat{n}=0$, and $s \in \mathbf{Z}$, so that Eq. (1) is relevant. As a first step,

\footnotetext{
${ }^{4}$ Actually, the states $\left| \pm \varpi_{0}\right\rangle,\left\langle \pm \varpi_{0}\right|$ do not really exist in the Hilbert space defined by the simultaneous existence of the two free fields $\vartheta_{1}$ and $\vartheta_{2}$ 21]. However, the limits Eq. (18) do exist if the $\vartheta_{1}$ representation is used; see section 4.2 for further remarks.
} 
let us explain why naive charge conservation cannot be used when evaluating matrix elements of Eq. (11), in particular the ground state matrix element (17).

\subsection{Reflection invariance and broken charge conserva- tion}

One obvious observation is that the three point function relevant for the coupling of minimal matter to gravity is known [2] to require a negative number of screening operators, while Eq. (11) contains only positive screenings, and thus a naive evaluation would give a vanishing result in this context. However, it is also possible to see purely from internal consistency considerations that charge conservation cannot hold: Consider the general classical solution of the Liouville equation,

$$
\exp [-\varphi]=A^{\prime-1}(u) A^{2}(u) B^{\prime-1}(v)\left(1-\frac{B(v)}{A(u)}\right)^{2}
$$

where $A(u)$ and $B(v)$ are two arbitrary functions. In ref. 20 the important observation was made that after gauge-fixing the projectively acting SL(2) symmetry of Eq. (19),

$$
A \rightarrow \frac{a A+b}{c A+d}, \quad B \rightarrow \frac{a B+b}{c B+d}
$$

by demanding that $A$ and $B$ have diagonal monodromy (i.e. that they be periodic up to a multiplicative constant, leading to periodic free fields), there remains the discrete symmetryf $A \rightarrow-1 / A, B \rightarrow-1 / B$, which leads to the existence of two equivalent free fields $\vartheta_{1}, \vartheta_{2}$ instead of just one. It was shown in [20 that this symmetry extends to the quantum level, and that there is a quantum canonical transformation relating $\vartheta_{1}$ and $\vartheta_{2}$. Now certainly all observables of the theory (i.e. all functionals of the Liouville field) must be invariant under the exchange

$$
\vartheta_{1} \leftrightarrow \vartheta_{2},
$$

\footnotetext{
${ }^{5}$ For general Toda theories, this becomes the symmetry of the theory under the Weyl group for the corresponding Lie algebra 22] 223].
} 
as this exchange just represents a particular $S L(2)$ transformation. We will call it reflection symmetry in the following. Due to the relation

$$
\tilde{\varpi}=-\varpi
$$

between the zero modes of $\varpi_{1}$ and $\varpi_{2}$, the vertex operators $\widetilde{V}_{-J}^{(J)} \widetilde{S}^{n} \overline{\widetilde{V}}_{-J}^{(J)} \overline{\widetilde{S}}^{n}$ formed using $\vartheta_{2}$ have zero mode shifts exactly opposite to those formed from $\vartheta_{1}$. :

$$
\left[\varpi, \widetilde{V}_{-J}^{(J)} \widetilde{S}^{n} \overline{\widetilde{V}}_{-J}^{(J)} \widetilde{\widetilde{S}}^{n}\right]=+2 m \widetilde{V}_{-J}^{(J)} \widetilde{S}^{n} \overline{\widetilde{V}}_{-J}^{(J)} \widetilde{\widetilde{S}}^{n}
$$

Now as formally the zero mode shifts in Eq. (11) are given by $\Delta \varpi \equiv-2 m=$ $2 J, 2 J-1,2 J-2, \ldots .$, ( similarly for Eq. (12)) we see that for generic $J$, there is no overlap at all between the shifts predicted using the two representations! Thus we conclude that charge conservation must be broken. The only exception is the case when $2 J$ is positive integer[], where the sum in Eq. (11) truncates at $n=2 J$ and the two sets of shifts indeed coincide. For this particular case, the requirement of reflection invariance Eq. (21) was shown in ref. [8] to largely fix the function $T(\varpi)$ in Eqs. (11), (12): []

$$
T(\varpi)=\frac{\Gamma(1-\varpi h / \pi)\lfloor\varpi\rfloor}{\Gamma(1+\varpi)}
$$

with the freedom of replacing $T(\varpi) \rightarrow T(\varpi) T_{1}(\varpi)$, where

$$
\frac{T_{1}(\varpi) T_{1}(-\varpi-2(n-J))}{T_{1}(\varpi+2(n-J)) T_{1}(-\varpi)}=1
$$

for any allowed value of $n$ and $2 J$. We will use Eq. (24) as an ansatz also for general $2 J$. 9

${ }^{6}$ We warn the reader familiar with the previous papers that the $\widetilde{V}$ operators here are different from those of [14] or [8]. There should be no confusion as the latter do not appear in the present paper.

${ }^{7}$ This is of course the case where the Liouville exponentials are in Kac's table, corresponding to the well-understood degenerate sector of Liouville theory [1] [14.

${ }^{8}$ Compared to ref. [8], we have replaced a factor $\sqrt{\lfloor\varpi\rfloor}$ by $\lfloor\varpi\rfloor$, making use of the freedom Eq. (25) below.

9 Actually, $T(\varpi)$ should be independent of $J$ in order to really drop out of the locality analysis. 
Returning to the general case, how does the theory manage to break charge conservation? The answer is that the infinite expansions Eq. (1) and Eq. (12) have rather subtle convergence properties (as was remarked already in ref. [8]), and must be resummed and regularized in an appropriate way to become truly well-defined. In particular, within matrix elements of the Liouville exponentials it is not allowed to permute the order of zero mode integration and sum over $n$ or $n, \widehat{n}$, which would lead to the naive charge conservation rules. For better readability, let us first sketch the main idea and then give a more exact technical derivation.

\subsection{Resummation and screening eigenstates}

The basic idea in order to render matrix elements of Eq. (11) well-defined is to insert a particular complete set of intermediate states, and then to carry out the sum over $n$ before the factorization sum. This complete set will then have to be chosen such that the $n$ sum can be carried out explicitly; now since Eq. (11) is an expansion in powers of screenings, it is natural to consider a basis composed of eigenstates of the latter. Thus we are lead to replace the matrix element (17) by

$$
\left\langle\varpi_{f}\left|\exp \left[-J \alpha_{-} \Phi\right]\right| \varpi_{i}\right\rangle \quad \rightarrow \quad \sum_{f} \int d y\left\langle\varpi_{f}\left|\exp \left[-J \alpha_{-} \Phi\right]\right| y ; f\right\rangle\left\langle y ; f \mid \varpi_{i}\right\rangle
$$

where $|y ; f\rangle$ denotes an eigenstate of the screening operator $S \bar{S}(\tau=\sigma=0) \equiv$ $S \bar{S}$, with eigenvalue parametrized by $y$ and $f$ a quantum number describing a basis of the corresponding eigenspace, and $\sum_{f} \int d y|y ; f\rangle\langle y ; f|=\mathbf{1}$. For fixed $y$, every term of the $n$ - sum in Eq. (四) contributes and the result will be given in terms of a $q$-hypergeometric function at $|q|=1$. The latter is well defined in the case where $2 J$ is integer - this is the origin of the restriction on one of the spins mentioned in the introduction - and can then be integrated over $y$ to give an exact closed form expression for the matrix element (17). It is invariant under the reflection symmetry mentioned above.

To make the above statements precise, let us first define eigenstates of

\footnotetext{
${ }^{10}$ Due to the symmetry of the three point function under permutation of the legs, our result will be valid also when one of the spins $2 J_{f}, 2 J_{i}$ corresponding to $\varpi_{f}$ and $\varpi_{i}$, rather than $2 J$, is integer.
} 
$S \bar{S}$ by

$$
\left|y ; f_{\varpi_{1}}\right\rangle:=\sum_{n=-\infty}^{\infty} e^{-i y n}\left(S_{0} \bar{S}_{0}\right)^{n}\left|f_{\varpi_{1}}\right\rangle
$$

In Eq. (27), $S_{0}, \bar{S}_{0}$ denote normalized screening charges with the property

$$
\left(S_{0} \bar{S}_{0}\right)^{\dagger}=\left(S_{0} \bar{S}_{0}\right)^{-1}
$$

Here $e^{i y}$ with $y \in[0,2 \pi]$ is the eigenvalue, and $f_{\varpi_{1}}$ denotes an arbitrary Fock state over the ground state $\left|\varpi_{1}\right\rangle$. The value of $\varpi_{1}$ will be fixed (up to integers) by the matrix element (17) under consideration. Of course the unitarity property of $S_{0} \bar{S}_{0}$ is needed for the eigenvalues $e^{i y}$ to be pure phases and hence for the sum in Eq. (27) to make sense. The latter is completely analogous to the formation of position eigenstates by a linear superposition of plane waves -the standard Fourier representation $\sum_{n=-\infty}^{\infty} e^{2 \pi i n\left(x-x_{0}\right)}$ of the (periodic) position space $\delta$ function. The normalized screening operator $S_{0} \bar{S}_{0}$ is related to $S \bar{S}$ by

$$
S_{0} \bar{S}_{0}=\frac{1}{\left(2 \pi \Gamma\left(1+\frac{h}{\pi}\right)\right)^{2}} \frac{h(\varpi+1)}{\sin h(\varpi+1)} \Gamma\left(1-\varpi \frac{h}{\pi}\right) \Gamma\left(1+(\varpi+2) \frac{h}{\pi}\right) S \bar{S}
$$

To check that Eq. (28) is fulfilled with this definition, one first observes that due to the hermiticity properties of the two free fields in the elliptic sector, $\vartheta_{1}^{\dagger}=\vartheta_{2}[20]$, one has

$$
(S \bar{S})^{\dagger}=\widetilde{S} \overline{\widetilde{S}}
$$

Thus if we write

$$
S_{0} \bar{S}_{0}=N_{1}(\varpi) S \bar{S}
$$

the unitarity requirement for $S_{0} \bar{S}_{0}$ becomes

$$
\frac{1}{\left|N_{1}(\varpi)\right|^{2}}=S \widetilde{S} \bar{S} \overline{\widetilde{S}}
$$

Now one has to compute $S \widetilde{S} \bar{S} \overline{\widetilde{S}}$. Since $\widetilde{S}$ has a zero mode shift opposite of $S$, we can identify it as a negative screening operator. Due to the reflection symmetry Eq. (21), positive and negative screenings must enter into the theory on exactly the same footing, and the latter, according to the above, possess a rigorous definition within the Gervais-Neveu quantization scheme 
without the need for any analytic continuation. The product $S \widetilde{S}$, which can be shown to exist without renormalization $\Pi$, is a dimension zero operator with vanishing zero mode shift. As these two data characterize a primary field in the free field Hilbert space up to an $\varpi$-dependent normalization [24], we conclude that

$$
S \widetilde{S}=K_{1}(\varpi) \mathbf{1}
$$

and thus Eq. (32) makes sense. As classically $S(u) \sim A(u)$ [13, the classical limit of Eq. (33) is simply the statement $A \cdot(-1 / A)=-1$. The factor $K_{1}(\varpi)$ is now easily computed from the associativity of the operator product, applied to the expression $V_{-\frac{1}{2}}^{\left(\frac{1}{2}\right)}(\sigma) S(\sigma) \widetilde{S}(\sigma)$. According to Eq. (15) we have

$$
V_{-\frac{1}{2}}^{\left(\frac{1}{2}\right)} S=I_{\frac{1}{2}}^{\left(\frac{1}{2}\right)}(\varpi) V_{+\frac{1}{2}}^{\left(\frac{1}{2}\right)}=I_{\frac{1}{2}}^{\left(\frac{1}{2}\right)}(\varpi) \widetilde{V_{-\frac{1}{2}}^{\left(\frac{1}{2}\right)}}
$$

where the last equality follows again from Eq. (23). Now $\widetilde{V_{-\frac{1}{2}}^{\left(\frac{1}{2}\right)}} \tilde{S}=$ $I_{\frac{1}{2}}^{\left(\widetilde{\left.\frac{1}{2}\right)}\right.}(\varpi) \widetilde{V_{\frac{1}{2}}^{\left(\frac{1}{2}\right)}}$ and thus, using Eq. (22),

$$
V_{-\frac{1}{2}}^{\left(\frac{1}{2}\right)}(\sigma) S(\sigma) \widetilde{S}(\sigma)=I_{\frac{1}{2}}^{\left(\frac{1}{2}\right)}(\varpi) I_{\frac{1}{2}}^{\left(\frac{1}{2}\right)}(-\varpi) V_{-\frac{1}{2}}^{\left(\frac{1}{2}\right)}(\sigma)
$$

from which we obtain immediately

$$
K_{1}(\varpi)=I_{\frac{1}{2}}^{\left(\frac{1}{2}\right)}(\varpi+1) I_{\frac{1}{2}}^{\left(\frac{1}{2}\right)}(-\varpi-1)
$$

Explicit computation now shows that $1 / K_{1}(\varpi)$ agrees with $N_{1}(\varpi)$ given by Eqs. (31),(29) and therefore Eq. (32) is fulfilled. It is immediate to generalize to $S^{n} S^{n}=K_{n}(\varpi) \mathbf{1} \equiv N_{n}^{-1}(\varpi) \mathbf{1}$ and we get

$$
\left(S_{0} \bar{S}_{0}\right)^{n}=N_{n}(\varpi)(S \bar{S})^{n}, \quad N_{n}(\varpi)=\prod_{l=1}^{n} N_{1}(\varpi+2(l-1))
$$

$N_{n}(\varpi)$ can be connected with the normalizations $I_{m}^{(J)}(\varpi)$ : Since we have the relations

$$
\widetilde{S}^{n}=I_{n}^{(0)}(-\varpi) V_{-n}^{(0)}
$$

\footnotetext{
${ }^{11}$ This can be understood as a consequence of the fact that the singular part of $\vartheta_{1}(u) \vartheta_{2}\left(u^{\prime}\right)$ is the same as that of $\vartheta_{1}(u) \vartheta_{1}\left(u^{\prime}\right)$ [20].
} 


$$
S^{-n}=I_{-n}^{(0)}(\varpi) V_{-n}^{(0)}
$$

we can also write

$$
N_{n}(\varpi)=\frac{I_{-n}^{(0)}(-\varpi)}{I_{n}^{(0)}(\varpi)}
$$

Comparing with Eq. (37), it is then checked immediately that $I_{-n}^{(0)}(\varpi)$ is given by the standard analytic continuation prescription $\prod_{l=1}^{-n} f(l):=1 / \prod_{l=1}^{n} f(l-$ $n)$; more generally one can show that the same is true for $I_{m}^{(J)}$ with $n=$ $J+m<0$.

From the states $\left|y ; f_{\varpi_{1}}\right\rangle$ we can now form the projector onto a fixed eigenvalue $e^{i y}$,

$$
P_{y}:=\frac{1}{2 \pi} \sum_{f_{\varpi_{1}}}\left|y ; f_{\varpi_{1}}\right\rangle\left\langle y ; f_{\varpi_{1}}\right|
$$

where the sum goes over all Fock states over the ground state $\left|\varpi_{1}\right\rangle$. The most important property of $P_{y}$ is the explicit presence of negative screenings, which will turn out not to decouple in the three point function when $P_{y}$ is inserted according to Eq. (26).

A remark about normalization is in order here. Since we consider only zero mode momenta which differ from some given starting value $\varpi_{i}$ by integers ${ }^{\mathbb{2}}$, we adopt the scalar product

$$
\left\langle\varpi^{\prime} \mid \varpi\right\rangle=\delta_{\varpi, \varpi^{\prime}}
$$

appropriate for discrete spectra. With this convention, we have, if $\varpi_{1}$ is taken to agree with $\varpi_{i}$ modulo an even integer, $\int_{0}^{2 \pi} d y P_{y}=1$ in the space $\cup_{l \in \mathbf{Z}} V_{l}$, the union of all Verma modules $V_{l}$ over the ground states $\varpi_{i}+2 l$, with $l$ an arbitrary integer. The projector $P_{y}$ thus works fine for the truncated theory living on a discrete spectrum over some arbitrary starting value $\varpi_{i}$. On the other hand, the full Liouville theory has a continous spectrum and hence the zero mode volume is infinite. Thus in order to compare with the DOZZ result at the particular integer points where it is possible, we will have to renormalize our three point function by the infinite volume of the zero mode integration; we will return to this point later.

\footnotetext{
${ }^{12}$ (when $2 J$ is integer, see below)
} 


\subsection{The three point function}

We are now in a position to write an explicit expression for the three-point matrix element (17) for the case where $\alpha_{+}$screenings are absent. Inserting the projector $P_{y}$ according to (26), we have

$$
P_{y}\left|\varpi_{i}\right\rangle=\frac{1}{2 \pi} \sum_{l=-\infty}^{\infty} e^{i y\left(\frac{\varpi_{1}-\varpi_{i}}{2}-l\right)}\left(S_{0} \bar{S}_{0}\right)^{l-\frac{\varpi_{1}-\varpi_{i}}{2}}\left|\varpi_{i}\right\rangle
$$

whence

$$
\begin{gathered}
\left\langle\varpi_{f}\left|\exp \left[-J \alpha_{-} \Phi\right](\tau=\sigma=0) P_{y}\right| \varpi_{i}\right\rangle=\sum_{n=0}^{\infty} \frac{T\left(\varpi_{f}\right)}{T\left(\varpi_{f}+2 n-2 J\right)} \\
\times a_{n}^{(J)}\left(\varpi_{f}\right) e^{i y(n-s)}\left\langle\varpi_{f}\left|V_{-J}^{(J)} S^{n} S_{0}{ }^{s-n} \bar{V}_{-J}^{(J)} \bar{S}^{n} \bar{S}_{0}{ }^{s-n}\right| \varpi_{i}\right\rangle .
\end{gathered}
$$

where $s=J-\frac{1}{2}\left(\varpi_{f}-\varpi_{i}\right)$ is the screening number of Eq. (10) and we have used Eq. (8) and Eq. (41) to reduce the $l$ sum from Eq. (42) to a single term. Writing $\left(S_{0} \bar{S}_{0}\right)^{n}=N_{n}(\varpi)(S \bar{S})^{n}$ as in the previous subsection, the matrix element of the Coulomb gas vertex operators is just

$$
\left\langle\varpi_{f}\left|V_{-J}^{(J)} S^{n} S_{0}{ }^{s-n} \bar{V}_{-J}^{(J)} \bar{S}^{n} \bar{S}_{0}{ }^{s-n}\right| \varpi_{i}\right\rangle=N_{s-n}\left(\varpi_{f}+2 n-2 J\right)\left|I_{s-J}^{(J)}\left(\varpi_{f}\right)\right|^{2}
$$

according to Eq. (15). Evaluating $a_{n}^{(J)}\left(\varpi_{f}\right) N_{s-n}\left(\varpi_{f}+2 n-2 J\right)$ explicitly, we thus obtain

$$
\begin{gathered}
\left\langle\varpi_{f}\left|\exp \left[-J \alpha_{-} \Phi\right] P_{y}\right| \varpi_{i}\right\rangle=\left|I_{s-J}^{(J)}\left(\varpi_{f}\right)\right|^{2}\left(\frac{\Gamma(1-h / \pi)}{2 \pi}\right)^{2 s} e^{-i s y} \\
\times \sum_{n=0}^{\infty} \frac{T\left(\varpi_{f}\right)}{T\left(\varpi_{f}+2 n-2 J\right)} \frac{\Gamma\left(\varpi_{i} h / \pi\right)}{\left.\Gamma\left[\left(\varpi_{f}+2 n-2 J\right) h / \pi\right)\right]\left(\varpi_{i}+1\right)_{n+2 s}} \\
\times(-1)^{n}\left(\begin{array}{c}
2 J \\
n
\end{array}\right)_{q} \frac{\left\lfloor\varpi_{i}\right\rfloor_{n+2 s}}{\left\lfloor\varpi_{f}+1\right\rfloor_{n}} e^{i n y}
\end{gathered}
$$

Here $\left(\begin{array}{c}2 J \\ n\end{array}\right)_{q} \equiv \frac{\lfloor 2 J\rfloor !}{\lfloor 2 J-n ! ! n\rfloor !}$ denotes a $q$-binomial, defined for negative integer $2 J=-p$ by $\left(\begin{array}{c}2 J \\ n\end{array}\right)_{q}=\frac{\prod_{i=0}^{n-1}\lfloor-p-i\rfloor}{\lfloor n\rfloor !}$. Pochhammer symbols $(a)_{n}$ for negative $n$ 
are given by $(a)_{n}=(-1)^{n} /(1-a)_{-n}$, and likewise for their $q$ - equivalents. Eq. (45) may be rewritten

$$
\begin{gathered}
\left\langle\varpi_{f}\left|\exp \left[-J \alpha_{-} \Phi\right] P_{y}\right| \varpi_{i}\right\rangle=\frac{T\left(\varpi_{f}\right)}{T\left(\varpi_{f}-2 J\right)} N_{s}\left(\varpi_{f}-2 J\right)\left|I_{\frac{1}{2}\left(\varpi_{i}-\varpi_{f}\right)}^{(J)}\left(\varpi_{f}\right)\right|^{2} \\
\times e^{-i y s} F^{(q)}\left(-2 J, \varpi_{f}-2 J ; \varpi_{f}+1 ; z\right)
\end{gathered}
$$

with $z:=e^{i y}$. Here, $F^{(q)}(a, b ; c ; z):=\sum_{n=0}^{\infty} \frac{\lfloor a\rfloor_{n}\lfloor b\rfloor_{n}}{\lfloor c\rfloor_{n}\lfloor n ! !}$ is the q-hypergeometric function [25], with $\lfloor a\rfloor_{n}:=\lfloor a\rfloor \ldots\lfloor a+n-1\rfloor$. Now $F^{(q)}(a, b ; c ; z)$ is a priori well-defined only for $|q|<1$ and $|z|<1$, while here $|q|=|z|=1$. However, in the special case

$$
2 J \in \mathbf{Z}
$$

the situation is different. If $2 J$ is a nonnegative integer, the hypergeometric function reduces to a finite sum, corresponding to the fact that in this case, the sum in Eq. (1) truncates at $n=2 J$. We then recover the Liouville exponentials for the degenerate (Kac's table) subsector. Though this case is trivial from the present point of view, as no resummation is necessary, we list it for completeness:

$$
\begin{gathered}
\left\langle\exp \left[-J_{f} \alpha_{-} \Phi\right] \exp \left[-J \alpha_{-} \Phi\right] \exp \left[-J_{i} \alpha_{-} \Phi\right]\right\rangle^{(J \geq 0)}= \\
g_{J, x_{f}+m}^{x_{f}} \bar{g}_{J, x_{f}+m}^{x_{f}} \frac{T\left(-\varpi_{0}\right)}{T\left(\varpi_{0}\right)}
\end{gathered}
$$

Of course this result could have been read off directly from Eq. (1) using naive charge conservation rules. We now turn to the interesting case

$$
2 J=-p
$$

with $p$ a positive integer. In this case as well, the hypergeometric function extends to $|q|=1$ and $|z|=1$ (in fact to arbitrary $q$ and $z$ ) via the transformation formula [25]

$$
\begin{gathered}
F^{(q)}\left(p, p+\varpi_{f} ; 1+\varpi_{f} ; z\right)=\frac{1}{\left(q^{p-1}-q^{1-p} z\right)\left(q^{p-3}-q^{3-p} z\right) \ldots\left(q^{1-p}-q^{p-1} z\right)} \\
\times F^{(q)}\left(1-p, 1+\varpi_{f}-p ; 1+\varpi_{f} ; z\right)
\end{gathered}
$$


where the hypergeometric function on the right-hand side is again a finite sum. For the matrix element Eq. (46), the reflection symmetry Eq. (21), which takes $V_{-J}^{(J)} S^{n} \bar{V}_{-J}^{(J)} \bar{S}^{n} \rightarrow \widetilde{V}_{-J}^{(J)} \widetilde{S}^{n} \overline{\widetilde{V}}_{-J}^{(J)} \overline{\widetilde{S}}^{n}, \quad \varpi \rightarrow-\varpi$, is readily translated into the condition that the right-hand side of Eq. (45) must be invariant under $\varpi_{f, i} \rightarrow-\varpi_{f, i}, \quad y \rightarrow-y$. (Notice we demand reflection invariance of the exponential in the middle separately, and not only of the matrix element as a whole, which would be a triviality). It is now easy to check that reflection invariance indeed holds for any integer $2 J$ with the expression Eq. (24), at any nonsingular point $z$. To arrive at the three point matrix element Eq. (17), it remains to perform the $y$ - integration. Eq. (50) exhibits first order poles at

$$
z_{j}=q^{2 p-2-2 j}, \quad j=0, \ldots 2(p-1)
$$

for which we need a prescription. There are three natural possibilities, $y \rightarrow$ $y \pm i \epsilon$ and taking the Cauchy principal value, and we will discuss all of them. In all cases, the integral over $y$ or $z$ reduces to a sum over residues, and we have

$$
\left\langle\varpi_{f}\left|\exp \left[-J \alpha_{-} \Phi\right]\right| \varpi_{i}\right\rangle=\left|I_{\frac{1}{2}\left(\varpi_{i}-\varpi_{f}\right)}^{(J)}\left(\varpi_{f}\right)\right|^{2} K_{1}\left(\varpi_{f}\right) K_{2}\left(\varpi_{i}\right)\left(R_{0}+R_{1}\right)
$$

Here,

$$
\begin{gathered}
R_{1}=\delta \frac{\left(q-q^{-1}\right)^{-2(p-1)}}{\lfloor 2 p-2\rfloor !} q^{(1-p)(2 p-3)} \sum_{j=0}^{2 p-2} q^{(2 s+2)(1-p+j)+j(2 p-3)} \\
\times(-1)^{j+1}\left(\begin{array}{c}
2 p-2 \\
j
\end{array}\right)_{q} F^{(q)}\left(1-p, 1+\varpi_{f}-p ; 1+\varpi_{f} ; q^{2 p-2 j-2}\right)
\end{gathered}
$$

is the sum of the residues at $|z|=1$, with the factor $\delta$ equal to $\frac{1}{2}$ for the Cauchy principal value prescription, 0 for $y \rightarrow y+i \epsilon$, and 1 for $y \rightarrow y-i \epsilon$. Furthermore, $R_{0}$ is the contribution from the residue at $z=0$, i.e. the trivial charge-conserving term which appears only for $s \geq 0$. It takes the simple form

$$
R_{0}=\frac{\lfloor p\rfloor_{s}\left\lfloor\varpi_{f}+s+1\right\rfloor_{p-1}}{\lfloor s\rfloor !\left\lfloor 1+\varpi_{f}\right\rfloor_{p-1}} .
$$

Of course it could have been obtained also directly from Eq. (11), using naive charge conservation rules. 
$R_{1}$ can be largely simplified. Indeed, one easily derives the identity

$$
\begin{aligned}
\sum_{j=0}^{2 p-2} q^{-j(2 n-2 s-2 p+1)}(-1)^{j} & \left(\begin{array}{c}
2 p-2 \\
j
\end{array}\right)_{q}=q^{(1-p)(2 n-2 s-2 p+1)}\left(q-q^{-1}\right)^{2 p-2} \\
& \times \frac{\lfloor n-s-1\rfloor !}{\lfloor n-s-2 p+1\rfloor !}
\end{aligned}
$$

where $s=J-\frac{1}{2}\left(\varpi_{f}-\varpi_{i}\right)$ as above. Thus we have

$$
R_{1}=-\delta\left(\begin{array}{c}
-s-1 \\
2 p-2
\end{array}\right)_{q}{ }_{3} F_{2}^{(q)}\left(1-p, 1+\varpi_{f}-p,-s ; 1+\varpi_{f},-s-2 p+2 ; 1\right)
$$

Here and below, we define expressions which are ill-defined for integer values of $s$ by replacing $s \rightarrow s+\epsilon$ and taking the limit $\epsilon \rightarrow 0$. [3] Eq. (56) can be reduced to a simple product of $q$ - factorials again [26], viz.

$$
R_{1}=-\delta \frac{\lfloor 1+s\rfloor_{p-1}\left\lfloor\varpi_{f}+s+1\right\rfloor_{p-1}}{\lfloor p-1\rfloor !\left\lfloor 1+\varpi_{f}\right\rfloor_{p-1}} .
$$

Comparing with Eq. (54), we see that the only difference to $R_{0}$ is the replacement of the binomial coefficient $\lfloor-2 J\rfloor_{s} /\lfloor s\rfloor$ ! from the expansion Eq. (1) by $-\lfloor 1+s\rfloor_{-2 J-1} /\lfloor-2 J-1\rfloor$ !, which can be viewed as an analytic continuation of the former. For $s \geq 0$, we have

$$
\delta \cdot R_{0}=-R_{1}
$$

One can now check whether reflection invariance holds true for our result Eq. (52). In general, for the matrix element (17), reflection invariance is equivalent to the replacement

$$
\varpi_{f} \rightarrow-\varpi_{f}, \varpi_{i} \rightarrow-\varpi_{i}
$$

We first observe that for the Cauchy principal value prescription $\delta=\frac{1}{2}$, reflection invariance is indeed fulfilled, though in a rather peculiar way: Under the transformation (59), the contribution from $R_{1}$ behaves antisymmetrically,

\footnotetext{
${ }^{13}$ We introduce this convention just to avoid clumsy notation; there is no actual ambiguity in the calculation.
} 
while the one from $R_{0}$ simply disappears when we go from positive $s$ to negative $\tilde{s}=-p-s$ (cf. Eq. (10)). We can express the result very concisely in terms of the coupling constants $g_{J, x+m}^{x} \bar{g}_{J, x+m}^{x}$ of Eq. (14). Indeed, we have

$$
\left\langle\varpi_{f}\left|\exp \left[-J \alpha_{-} \Phi\right]\right| \varpi_{i}\right\rangle=\frac{1}{2} \cdot g_{J, x_{f}+m}^{x_{f}} \bar{g}_{J, x_{f}+m}^{x_{f}} \frac{T\left(\varpi_{f}\right)}{T\left(\varpi_{i}\right)}
$$

Eq. (60) needs some explanation in the case $n=J+m=s<0$, as the coupling constants corresponding to negative screenings were not derived in refs. [14, 8]. However, using the associativity of the operator product, they can be deduced from the couplings with $s \geq 0$ [27]; this will be worked out in detail elsewhere. We need here only the result that in the case $s<0$, the coupling constants are still given by Eq. (16), with $a_{n}^{(J)}$ and $I_{m}^{(J)}(\varpi)$ continued to negative $n=J+m$ as explained below Eq. (39), except that $\frac{\lfloor-2 J\rfloor_{n}}{\lfloor n\rfloor !}$ in Eq. (2) is replaced by $-\frac{\lfloor 1+n\rfloor_{p-1}}{\lfloor p-1\rfloor !}$. Invoking conformal invariance [24], we can promote the result Eq. (60) for the ground state matrix element to an operator equation giving an "effective" representation for the Liouville exponentials Eq. (1]):

$$
\exp \left[-J \alpha_{-} \Phi\right]_{\mathrm{eff}}=\sum_{n=-\infty}^{\infty} \frac{T(\varpi)}{T(\varpi+2 m)} \frac{1}{2} g_{J, x+m}^{x} \bar{g}_{J, x+m}^{x} V_{m}^{(J)} \bar{V}_{m}^{(J)}
$$

for $2 J=-p<0$. By definition, Eq. (61) is to be evaluated using naive charge conservation, in contrast to Eq. (1). It is Eq. (61) which should be viewed as the true chiral decomposition of the Liouville exponentials. Intriguingly, Eq. (61) can be reexpressed in terms of the original expansion Eq. (1) as follows:

$$
\begin{gathered}
\exp \left[-J \alpha_{-} \Phi\right]_{\text {eff }}=\frac{1}{2}\left(\sum_{n=0}^{\infty} \frac{T(\varpi)}{T(\varpi+2 m)} g_{J, x+m}^{x} \bar{g}_{J, x+m}^{x} V_{m}^{(J)} \bar{V}_{m}^{(J)}+\right. \\
\left.\sum_{\widetilde{n}=0}^{\infty} \frac{T(\widetilde{\varpi})}{T(\widetilde{\varpi}+2 \widetilde{m})} g_{J, \widetilde{x}+\widetilde{m}}^{\widetilde{g}} \bar{g}_{J, \widetilde{x}+\widetilde{m}}^{\widetilde{V}} \widetilde{\widetilde{m}}_{\widetilde{V}}^{(J)} \overline{\widetilde{V}}_{\widetilde{m}}^{(J)}\right)
\end{gathered}
$$

where $\widetilde{\varpi}=-\varpi, \varpi_{0}+2 \widetilde{x}=-\varpi$, and the $\widetilde{V}$ fields are formed from the free field $\vartheta_{2}$ as in Eq. (23). To show this, let us first observe that the sum over negative $n$ in Eq. (61) effectively starts only at $n \leq 2 J$ as the coefficients 
$g_{J_{1}, x+m_{1}}^{x} \bar{g}_{J_{1}, x+m_{1}}^{x}$ vanish for $2 J_{1}+1 \leq n_{1} \leq-1$. Now the reflection symmetry discussed above tells us that

$$
\frac{T(\varpi)}{T\left(\varpi+2 m_{1}\right)} g_{J_{1}, x+m_{1}}^{x} \bar{g}_{J_{1}, x+m_{1}}^{x}=\frac{T(-\varpi)}{T\left(-\varpi-2 m_{1}\right)} g_{J_{1}, \tilde{x}-m_{1}}^{\tilde{x}} \bar{g}_{J_{1}, \tilde{x}-m_{1}}^{\tilde{x}}
$$

The shift $-m_{1}=\widetilde{m}_{1}$ corresponds to $\tilde{n}_{1}=J_{1}-m_{1}=2 J_{1}-n_{1} \geq 0$ and so we recover the coefficients of the $n_{1} \geq 0$ part, evaluated at $-\varpi=\widetilde{\varpi}$. Furthermore, we have $V_{m_{1}}^{\left(J_{1}\right.}=\widetilde{V}_{-m_{1}}^{\left(J_{1}\right)}$ and so indeed the $n<0$ summation in Eq. (61) has the same form as the $n \geq 0$ part with $\vartheta_{1}$ replaced by $\vartheta_{2}$. Thus, the whole effect of the complicated analysis above is to symmetrize Eq. (11) in terms of the $\vartheta_{1}$ and $\vartheta_{2}$ representations." Of course, this apparent simplicity is somewhat deceiving as the relation between the two free fields is very complicated, and it is by no means trivial to obtain higher point correlation functions from Eq. (61) or Eq. (62).

Let us now turn to the second possibility for treating the residues. The $i \epsilon$ - prescription is consistent with reflection invariance only if we employ the $s$-dependent prescription $y \rightarrow y+i \epsilon \operatorname{sgn} s$, with $\operatorname{sgn} s:=1$ for $s \geq 0$, and -1 otherwise. In this case, there is a residue contribution only for $s<0$ and one obtains

$$
\begin{aligned}
& \left\langle\varpi_{f}\left|\exp \left[-J \alpha_{-} \Phi\right]\right| \varpi_{i}\right\rangle= \\
& 1 \cdot g_{J, x_{f}+m}^{x_{f}} \bar{g}_{J, x_{f}+m}^{x_{f}} \frac{T\left(\varpi_{f}\right)}{T\left(\varpi_{i}\right)}
\end{aligned}
$$

and similarly in place of Eq. (61). Thus the two choices differ only by an overall normalization factor. Within the discrete spectrum/finite zero mode volume theory considered here, the proper normalization can be determined by looking at the operator product expansion of two Liouville exponentials. This will be done in the next paragraph, and one finds that the choice Eq. (60) is preferred over Eq. (64).

Eq. (61) holds for $2 J<0$; in the case $2 J \geq 0$ where the sum in Eq. (1) is finite and we have only charge-conserving terms, the effective representation of course coincides with Eq. (1) itself, or Eq. (14). We can unify both cases through the formula Eq. (62), which trivially holds also when $2 J \geq 0$ (cf. section 3.1).

\footnotetext{
${ }^{14}$ The picture will however be a little bit more complicated in the two screening case to be discussed below.
} 
We can now write down the full three point function, making use of the identification Eq. (18). For this we still need the normalization factors $F_{i}, F_{f}$ in Eq. (18), which are easily determined from Eqs. (2), (24) and $\lim _{\tau \rightarrow-\infty}(z \bar{z})^{-\Delta_{J}} V_{-J}^{(J)} \bar{V}_{-J}^{(J)}(\sigma,-i \tau)\left|\varpi_{0}\right\rangle=1\left|\varpi_{J}\right\rangle$, with $\varpi_{J}=\varpi_{0}+2 J \equiv 1+$ $\pi / h+2 J$, and similarly for the left external state. For integer $2 J_{i}, 2 J_{f}$ we have the simple relations

$$
\begin{gathered}
F_{i}=\epsilon_{i} \frac{T\left(\varpi_{i}\right)}{T\left(\varpi_{0}\right)} \\
F_{f}=\epsilon_{f} \frac{T\left(-\varpi_{0}\right)}{T\left(\varpi_{f}\right)}
\end{gathered}
$$

with $\epsilon_{i, f}=1$ for $2 J_{i, f} \geq 0$, and $\epsilon_{i, f}=\frac{1}{2}$ otherwise, according to Eq. (61). We would like to stress here that there is no restriction on $\varpi_{i}, \varpi_{f}$ in Eq. (60) other than Eq. (10), so that up to this point, $\varpi_{f}, \varpi_{i}$ can take continous values. It is only when we try to represent the states $\left|\varpi_{i, f}\right\rangle$ in terms of Liouville exponentials that we have to respect the constraint Eq. (47) arising in our quantum treatment of the latter. Combining Eqs. (65), (18) and (60), we obtain

$$
\left\langle\exp \left[-J_{f} \alpha_{-} \Phi\right] \exp \left[-J \alpha_{-} \Phi\right] \exp \left[-J_{i} \alpha_{-} \Phi\right]\right\rangle=\epsilon_{i} \epsilon_{f} \epsilon_{J} \frac{T\left(-\varpi_{0}\right)}{T\left(\varpi_{0}\right)} g_{J, x+m}^{x} \bar{g}_{J, x+m}^{x}
$$

We have not yet discussed the dependence on the cosmological constant; likewise, we did not fix the overall $c$ - number normalization freedom

$$
\exp \left[-J \alpha_{-} \Phi\right] \rightarrow c_{J} \exp \left[-J \alpha_{-} \Phi\right]
$$

which is left over by the locality analysis. In refs. [28], [8] it was observed that the ambiguity (67) can be fixed by imposing the leading order multiplicativity property $\exp \left[-J \alpha_{-} \Phi\right] \exp \left[-J^{\prime} \alpha_{-} \Phi\right] \sim \exp \left[-\left(J+J^{\prime}\right) \alpha_{-} \Phi\right]$ and the quantum equations of motion to be $c_{J}=c^{J}$, with

$$
c=\frac{\pi \sin h}{8 h^{2}} \Gamma^{2}(1+h / \pi)
$$

Reinstating the cosmological constant provides another factor $\left(\mu^{2}\right)^{J}$, so that the correctly normalized three point function finally becomes

$$
\left\langle\exp \left[-J_{f} \alpha_{-} \Phi\right] \exp \left[-J \alpha_{-} \Phi\right] \exp \left[-J_{i} \alpha_{-} \Phi\right]\right\rangle_{\mu^{2}}=
$$




$$
\left(\mu^{2} \frac{\pi \sin h}{8 h^{2}} \Gamma^{2}(1+h / \pi)\right)^{J+J_{i}+J_{f}+\varpi_{0}}\left\langle\exp \left[-J_{f} \alpha_{-} \Phi\right] \exp \left[-J \alpha_{-} \Phi\right] \exp \left[-J_{i} \alpha_{-} \Phi\right]\right\rangle
$$

where we have added a contribution $\varpi_{0}$ to the exponent to account for the contribution of the background charge to the cosmological constant dependence [29].

\subsection{Operator product with a degenerate field}

Due to the infinite sum in Eq. (61), the operator product of two general exponentials is rather delicate. In the present analysis, we will restrict ourselves to the case where one of them is degenerate, which in the single screening case means $2 J$ nonnegative integer. Then the operator product with an arbitrary conformal field is controlled by the null vector decoupling equations [24] [7] and the situation is much clearer. Consider therefore

$$
\exp \left[-J_{1} \alpha_{-} \Phi\right]_{\mathrm{eff}}(\tau, \sigma) \exp \left[-J_{2} \alpha_{-} \Phi\right]\left(\tau^{\prime}, \sigma^{\prime}\right)
$$

with $2 J_{1}=-p<0$ and $2 J_{2} \geq 0$. We take $e_{\text {eff }}^{-J_{1} \alpha_{-} \Phi}$ to be given by Eq. (62), except that the factor $\frac{1}{2}$ is replaced by a general normalization $\delta_{J_{1}}$ to be determined (cf. the remark below Eq. (64)), i.e. $e_{\text {eff }}^{-J_{1} \alpha_{-} \Phi}=\delta_{J_{1}}\left(e_{\vartheta_{1}}^{-J_{1} \alpha_{-} \Phi}+\right.$ $\left.e_{\vartheta_{2}}^{-J_{1} \alpha_{-} \Phi}\right)$. For the above operator product, the triangular inequalities [14]

$$
-\left|J_{1}-J_{2}\right| \leq J_{12} \leq J_{1}+J_{2}
$$

are valid, and so only a finite number of Liouville exponentials $e^{-J_{12} \alpha_{-} \Phi}$ will appear on the right hand side. As a side remark, we note that the leading short-distance singularity in the product is given by $J_{12}=J_{1}+J_{2}$ as expected if and only if

$$
J_{1}-J_{2} \geq-\varpi_{0}
$$

For $J_{2}=-J_{1}$, this becomes the Seiberg bound [9] for $J_{1}$ ! We leave the significance of this observation to further study. Let us now first consider the contribution to $e_{\text {eff }}^{-J_{1} \alpha_{-} \Phi}$ from the $\vartheta_{1}$ - representation. For this part, the fusion was already written in ref. [8] (within the coordinates of the sphere)

$$
\exp \left[-J_{1} \alpha_{-} \Phi\right]_{\vartheta_{1}}\left(z_{1}, \bar{z}_{1}\right) \exp \left[-J_{2} \alpha_{-} \Phi\right]\left(z_{2}, \bar{z}_{2}\right)=
$$

\footnotetext{
${ }^{15}$ We suppress the usual short-distance factor.
} 


$$
\begin{gathered}
\sum_{J_{12}} \sum_{\{\nu\},\{\bar{\nu}\}} \exp \left[-J_{12} \alpha_{-} \Phi^{\{\nu\},\{\bar{\nu}\}}\right]_{\vartheta_{1}}\left(z_{2}, \bar{z}_{2}\right) \times \\
<\varpi_{J_{12}} ;\{\nu\},\{\bar{\nu}\}\left|\exp \left[-J_{1} \alpha_{-} \Phi\right]_{\vartheta_{1}}\left(z_{1}-z_{2}, \bar{z}_{1}-\bar{z}_{2}\right)\right| \varpi_{J_{2}}>.
\end{gathered}
$$

Here, $\{\nu\},\{\bar{\nu}\}$ denote arbitrary descendants, and $\varpi_{J}=\varpi_{0}+2 J$. Eq. (72), which was derived from the chiral fusion algebra, is a priori valid only on a formal level as the Liouville exponentials were treated as formal power series in screening charges in ref. [8]. Indeed, for general $J_{1}, J_{2}$ there is no truncation condition cutting off large negative $J_{12}$ so that infinite short-distance singularities would arise in Eq. (72). However, if at least one of the exponentials is degenerate so that the inequalities (70) hold this problem is absent and Eq. (72) is valid as a consequence of the null vector decoupling equations. Let us now turn to the $\vartheta_{2}$ - contribution. The Liouville exponentials with $2 J \geq 0$ are already manifestly reflection invariant [8] (the replacement $\vartheta_{1} \rightarrow \vartheta_{2}$ amounts only to a renumbering of terms), and so we can trivially substitute $\vartheta_{2}$ for $\vartheta_{1}$ in $e^{-J_{2} \alpha_{-} \Phi}$. Then of course Eq. (72) is again valid for the operator product, with the obvious replacements, and the two contributions add up to form a symmetrized outgoing exponential in the form Eq. (62) again

$$
\begin{gathered}
\exp \left[-J_{1} \alpha_{-} \Phi\right]_{\operatorname{eff}}\left(z_{1}, \bar{z}_{1}\right) \exp \left[-J_{2} \alpha_{-} \Phi\right]\left(z_{2}, \bar{z}_{2}\right)= \\
\rho_{J_{12}} \sum_{J_{12}} \sum_{\{\nu\},\{\bar{\nu}\}} \exp \left[-J_{12} \alpha_{-} \Phi^{\{\nu\},\{\bar{\nu}\}}\right] \operatorname{eff}\left(z_{2}, \bar{z}_{2}\right) \times \\
<\varpi_{J_{12}} ;\{\nu\},\{\bar{\nu}\}\left|\exp \left[-J_{1} \alpha_{-} \Phi\right]_{\vartheta_{1}}\left(z_{1}-z_{2}, \bar{z}_{1}-\bar{z}_{2}\right)\right| \varpi_{J_{2}}>.
\end{gathered}
$$

with $\rho_{J_{12}}=2 \delta_{J_{1}}$ for $J_{12} \geq 0$ where the Liouville exponentials are known to have unit normalization, and $\rho_{J_{12}}=\delta_{J_{1}} / \delta_{J_{12}}$ for $J_{12}<0$. Let us consider the case $J_{2}=-J_{1}=-J$ now. To leading order, we expect the relation

$$
\exp \left[-J \alpha_{-} \Phi\right](\tau, \sigma) \exp \left[+J \alpha_{-} \Phi\right]\left(\tau^{\prime}, \sigma^{\prime}\right) \sim \mathbf{1}
$$

On the other hand, Eq. (72) gives

$$
\exp \left[-J \alpha_{-} \Phi\right](\tau, \sigma) \exp \left[+J \alpha_{-} \Phi\right]\left(\tau^{\prime}, \sigma^{\prime}\right) \sim 2 \delta_{J} \mathbf{1}
$$

\footnotetext{
${ }^{16}$ The exchange $\vartheta_{1} \leftrightarrow \vartheta_{2}$ in the matrix element on the right hand side of Eq. (72) amounts only to a trivial renaming of variables.
} 
and so we conclude that $\delta_{J}=\frac{1}{2}$ as in Eqs. (61) and (62), and $\rho_{J_{12}}=1$ in all cases.

We close this section with a few remarks on locality. The locality properties of the Liouville exponentials in the form Eq. (1) or Eq. (12) were analyzed in great detail in ref. [8]. However, one may wonder whether this analysis is affected by the new nonperturbative contributions derived above for $J<0$. We have seen that in the region $s=n \geq 0$, the only effect of the residue contribution is a renormalization of the coefficients in Eq. (臬) by a factor $\frac{1}{2}$. On the other hand, the positive and negative powers of screenings remain of the same type under braiding [27] and so the mutual locality of the positive screening parts of the exponentials as analyzed in ref. [8] is certainly a necessary condition for locality. Reflection invariance then guarantees that also the negative screening contributions are mutually local. On the other hand, one still has to check the relative locality of positive and negative screening contributions; this will be shown elsewhere within a detailed analysis of the fusion and braiding symbols for negative screenings. At this point, we would like to make contact with ref. [16], where recursion relations for the Liouville three point function are derived by exploiting crossing symmetry for a four point function with one degenerate ( $\operatorname{spin} \frac{1}{2}$ ) exponential. As explained in ref. [15], these are just the same recursion relations as those fulfilled by the coupling constants $g_{J, x+m}^{x} \bar{g}_{J, x+m}^{x}$ of the chiral operator product algebra (for the latter, they were derived in ref. [14)Ш. In fact the crossing symmetry conditions as analyzed in [16] are equivalent to demanding that $\left[e^{-\frac{1}{2} \alpha_{ \pm} \Phi}(\tau, \sigma), e^{-J \alpha_{-} \Phi}\left(\tau, \sigma^{\prime}\right)\right]=0$, that is, locality between the spin $\frac{1}{2}$ exponential with both screening types and any other one with arbitrary spin $J$. As this problem is completely controlled by the null vector decoupling equations obeyed by $e^{-\frac{1}{2} \alpha_{ \pm} \Phi}$ [24], for this particular situation one automatically obtains the exact nonperturbative locality conditions. Remarkably, these conditions, although they pertain only to a very particular case, are stringent enough to determine the most general three point couplings under the assumption that the latter are continous in the spins; this is because the set of shifts $\Delta J=n+\widehat{n} \frac{\alpha_{+}}{\alpha_{-}}$lies dense in the real numbers provided $\alpha_{+}, \alpha_{-}$are incommensurate, which is the case for generic values of the central charge. However in the truncated theory considered here, where only integer $2 J$ and

\footnotetext{
${ }^{17}$ (within the special context of positive half-integer spins, but they can be immediately extended - see ref. [15]).
} 
$s$ are admitted, this argument works only within the separate regions $2 J \geq 0$ and $2 J<0$, or $s>0$ and $s \leq 0$, as the ratio of the coefficients at the boundary is either zero or ill-defined. As the same functional form of the coefficients governs both regions $2 J \geq 0$ (where locality is known to hold as a consequence of the null vector decoupling equations [24] [7]), and $2 J<0$, it is not surprising that the above crossing symmetry recursion relations are indeed fulfilled also by the coefficients in Eq. (61) in the region $2 J<0$; a more detailed exposition of this point can be found in ref. [15].

\section{The principle of monodromy invariance and the extension to the two screening case}

To explain the basic idea, let us first consider the classical theory. Eq. (19) gives for general Liouville exponentials

$$
\begin{gathered}
\exp [-J \varphi]=\left(A A^{\prime-\frac{1}{2}} B^{\prime-\frac{1}{2}}\right)^{2 J}\left(1-\frac{B}{A}\right)^{2 J}= \\
\left(A A^{\prime-\frac{1}{2}} B^{\prime-\frac{1}{2}}\right)^{2 J} \sum_{n=0}^{\infty}\left(\begin{array}{c}
2 J \\
n
\end{array}\right)(-1)^{n}\left(\frac{B}{A}\right)^{n} .
\end{gathered}
$$

$B / A$ is just the classical version of the screening operator $S \bar{S}$ while $\left(A A^{\prime-\frac{1}{2}} B^{\prime-\frac{1}{2}}\right)^{2 J}$ corresponds to $V_{-J}^{(J)} \bar{V}_{-J}^{(J)}$, and Eq. (回) is the quantum version of of Eq. (76). In the elliptic sector, one has [20] $\left|\frac{B}{A}\right| \equiv 1$. There is a slight subtlety in the passage from Eq. (19) to Eq. (76) which turns out to have important consequences. Namely, Eq. (19) is single-valued (as it must be, since the classical Liouville field is real), while Eq. (76) is not ${ }^{18}$. Indeed, if $A$ and $B$ are reexpressed in terms of chiral free fields [20] [21], the dependence on the classical free field zero mode $q_{0}$ becomes $A A^{\prime-\frac{1}{2}} B^{\prime-\frac{1}{2}} \sim e^{-i q_{0}}$ and $\frac{B}{A} \sim e^{2 i q_{0}}$. Then under a rotation $q_{0} \rightarrow q_{0}+2 \pi$ around the unit circle, Eq. (76) picks up a factor $e^{2 \pi i J}$ (the binomial series is periodic term by term), while Eq. (19), and any power of it, is invariant. Consequently, when performing the zero mode integration in the quantum case within the evaluation of some matrix element, we are not entitled to use the expansion

\footnotetext{
${ }^{18}$ This was already noted in ref. [8] in the context of the periodicity properties of the Liouville exponentials.
} 
Eq. (11) throughout the full range $q_{0} \in(-\infty,+\infty)$, but only in some interval of length $2 \pi$. A remedy exactly appropriate for this problem was proposed in ref. [17] within the context of the calculation of conformal blocks for theories based on $S L(2)$ Kac-Moody current algebra. For pedagogical purposes, let us consider instead of Eq. (76) the simplified toy version

$$
f_{J}(z):=z^{-J}(1-z)^{2 J}
$$

with $|z|=1$. The nontrivial monodromy of $f_{J}(z)$ around the origin captures the essence of the non-singlevaluedness properties of Eq. (76) or Eq. (西). A three point correlator would then be modeled by the product $f_{J_{1}}(z) f_{J_{2}}(z) f_{J_{3}}(z)$. It was observed in [17 that on the unit circle, there is an infinity of equivalent representations of $f_{J}(z)$,

$$
f_{J}^{(\gamma)}(z)=z^{J} \sum_{n=-\infty}^{\infty}\left(\begin{array}{c}
2 J \\
n+\gamma
\end{array}\right) z^{n+\gamma}(-1)^{n} e^{i \pi \gamma}
$$

with $\gamma$ arbitrary real, which can be taken to be real and to agree with $f_{J}(z)$ within some fundamental interval for $\arg z$ (say $\arg z \in(-\pi, \pi)$ ), while differing from $f_{J}(z)$ by their monodromy around $z=0$. But then, if in a "three point function" $f_{J_{f}}^{\left(\gamma_{f}\right)}(z) f_{J}^{(\gamma)}(z) f_{J_{i}}^{\left(\gamma_{i}\right)}(z)$ we choose $\gamma_{i}, \gamma, \gamma_{f}$ such that $\gamma_{f}-J_{f}+\gamma-J+\gamma_{i}-J_{i}$ is integer, the total monodromy vanishes and the non-singlevaluedness problem goes away. This means that the expansion of the observables has to be chosen according to the correlator resp. matrix element under consideration. In the true quantum theory we would consider the expression $\left\langle\exp \left[-J_{f} \alpha_{-} \Phi\right] \exp \left[-J_{\alpha_{-}} \Phi\right] \exp \left[-J_{i} \alpha_{-} \Phi\right]\right\rangle$, or equivalently, (17), where the monodromy properties of the two "external" primaries have been encoded in the corresponding states according to Eq. (18) through the values of $\varpi_{f}, \varpi_{i}$. Using the expansion Eq. (11) for the exponentials corresponds to the case $\gamma_{f}=\gamma=\gamma_{i}=0$ above. Indeed, from Eq. (1) we infer that the total dependence on the quantum zero mode $q_{0}$ - rescaled by a factor $\alpha_{-}$with respect to the classical one [8] - is $\exp \left[q_{0} \alpha_{-}\left(J_{f}+J+J_{i}+\varpi_{0}+r\right)\right]$, where $r$ is an integer, and the term $\varpi_{0}$ counts the difference in the momenta of the left and right vacuum, which is given by the background charge. Thus, monodromy invariance requires $J_{f}+J+J_{i}+\varpi_{0}=0$ modulo integers, or $\frac{1}{2}\left(\varpi_{f}-\varpi_{i}\right)-J$ integer. In order to obtain more general three point functions, we should introduce expansions for the Liouville exponentials with nontrivial $\gamma$. However, according to Eq. (18), the monodromy parameters of the 
exterior states in the matrix element (17) are always fixed to be $\gamma_{i}=\gamma_{f}=0$. This is not a problem, as the full "monodromy deficit" can be absorbed by $\gamma$. Now general $\gamma$ - expansions of the quantum version of $\left(1-\frac{B}{A}\right)^{2 J}$ will involve arbitrary real powers $(S \bar{S})^{n+\gamma}$ of screenings - defined by taking their zero mode shifts to be $\Delta \varpi=-2(n+\gamma)$ - corresponding to general discrete representations of $U_{q}(\operatorname{sl}(2))$. The algebra of these objects is unknown to date, and also we cannot expect the modification of the coefficients $a_{n}^{(J)}(\varpi)$ to be given by the simple replacement $n \rightarrow n+\gamma$ as in the above toy example; rather it should follow from repeating the locality analysis of ref. [8] in this more general situation. However, there is an important special case where the solution is already known: When $\gamma_{f}+\gamma+\gamma_{i}=\gamma=\widehat{n} \frac{\pi}{h}$, we have $(S \bar{S})^{n+\gamma}=(S \bar{S})^{n+\widehat{n} \pi / h} \sim(S \bar{S})^{n}(\widehat{S} \widehat{\bar{S}})^{\widehat{n}}$ as is easily checked by comparing the zero mode shifts. For this situation, the locality analysis was already performed in ref. [8], and the result was precisely Eq. (12). Thus we are lead to identify Eq. (12) with the expansion of the Liouville exponentials carrying monodromies appropriate for matrix elements with

$$
\frac{1}{2}\left(\varpi_{f}-\varpi_{i}\right)-J=n+\widehat{n} \frac{\pi}{h} .
$$

with $n, \widehat{n}$ arbitrary integers. Let us now analyze the three point matrix element (17) in the general setting Eq. (79). There are two different points of view that we can take, which essentially lead to the same result. According to the discussion above, in the situation Eq. (79) where nontrivial monodromies are involved, we should actually consider instead of Eq. (12) or (14) an expansion of the form

$$
\exp \left[-J^{e} \alpha_{-} \Phi\right]_{\mathrm{eff}}=\delta_{J^{e}} \sum_{\underline{n}} \frac{T(\varpi)}{T(\varpi+2 m)} g_{J^{e}, x+m^{e}}^{x} \bar{g}_{J^{e}, x+m^{e}}^{x} V_{m^{e}}^{\left(J^{e}\right)} \bar{V}_{m^{e}}^{\left(J^{e}\right)}
$$

where the $n, \widehat{n}$ sums extend a priori over arbitrary integers. The values of the coupling constants outside the region $n, \widehat{n} \geq 0$ would then be determined directly from locality and we would arrive at an effective representation of the form Eq. (61) without performing a residue calculation. Of course, the relative normalizations of regions not connected by the locality conditions or reflection invariance cannot be fixed by this analysis. On the other hand, we can take Eq. (12) as a starting point for a nonperturbative analysis in the same way as we did for Eq. (1). We will briefly indicate how the calculation is carried out in this more general setting. 


\subsection{Residue calculation in the two screening case}

The logic of the derivation goes exactly as in section 3.3. Instead of Eq.(40), we introduce the projector

$$
P_{y, \hat{y}}:=\left(\frac{1}{2 \pi}\right)^{2} \sum_{f_{\varpi_{1}}}\left|y, \hat{y} ; f_{\varpi_{1}}\right\rangle\left\langle y, \hat{y} ; f_{\varpi_{1}}\right|
$$

formed from the eigenstates

$$
\left|y, \hat{y} ; f_{\varpi_{1}}\right\rangle:=\sum_{n, \widehat{n}=-\infty}^{\infty} e^{-i y n} e^{-i \hat{y} \widehat{n}}\left(S_{0} \bar{S}_{0}\right)^{n}\left(\widehat{S}_{0} \widehat{\bar{S}}_{0}\right)^{\widehat{n}}\left|f_{\varpi_{1}}\right\rangle
$$

with $\widehat{S}$ as in Eq. (12). Starting from the expansion Eq. (12), all steps in the above calculation can then be repeated, if we use that $\left[S_{0}, \widehat{S}_{0}\right]=0$ (this follows from $[S, \widehat{S}]=0$, which was shown in ref. [8]). The residue sums factorize into two expressions of the form Eq. (53) and Eq. (54), related by the exchange $h \leftrightarrow \widehat{h} \equiv \pi^{2} / h$. The restriction Eq. (47) is replaced by

$$
2 J^{e} \in \mathbf{Z}+\mathbf{Z} \frac{\pi}{h}
$$

and the screening numbers are subject to Eq. (11). We slightly changed the notation $J \rightarrow J^{e}$ to make it clear that $2 J$ need not be integer any more, and similarly we will write $s^{e}$ instead of $s$. It will be convenient to decompose

$$
J^{e}=J+\widehat{J} \frac{\pi}{h}, \quad s^{e}=s+\widehat{s} \frac{\pi}{h}
$$

with $s, \widehat{s}, 2 J, 2 \widehat{J}$ integer.19 The normalization constants $I_{m^{e}}^{\left(J^{e}\right)}$ (with $m^{e}=$ $n+\widehat{n} \frac{\pi}{h}$ ) in this more general situation have been computed in ref. [8] for $n, \widehat{n}$ positive (see appendix) and can immediately be continued to $n$ or $\widehat{n}$ negative using the argument given in the previous section.

The result can again be written in a form similar to Eq. (60), where residue contributions are relevant. There is a factor $\frac{1}{2}$ if only one of the spins $J, \widehat{J}$ is negative so that only one residue contribution is present, and a factor $\frac{1}{4}$ in the case $J<0, \widehat{J}<0$ where there are two. The coupling constants are now given by (cf. [14], [8])

$$
g_{J^{e}, x_{f}+m^{e}}^{x_{f}} \bar{g}_{J^{e}, x_{f}+m^{e}}^{x_{f}}=a_{n, \widehat{n}}^{\left(J^{e}\right)}(\varpi)\left|I_{m^{e}}^{\left(J^{e}\right)}(\varpi)\right|^{2}
$$

\footnotetext{
${ }^{19}$ The same notation is used in refs. 14, [8].
} 
for $J, \widehat{J} \geq 0$. If, say, $J<0$ but $\widehat{J} \geq 0$ one has to replace the factor $\frac{\lfloor-2 J\rfloor_{n}}{\lfloor n ! !}$ in $a_{n, \widehat{n}}^{\left(J^{e}\right)}$ by $-\frac{\left\lfloor 1+\left.s\right|_{p-1}\right.}{\lfloor p-1] !}$ just as below Eq. (60), and similarly for $J \geq 0, \widehat{J}<0$ and $J<0, \widehat{J}<0$. The calculation is well-defined everywhere except for the regions

$$
\begin{array}{ll}
J \geq 0, \widehat{J} \geq 0, & s \geq 2 J+1 \wedge \widehat{s} \geq 2 \widehat{J}+1 \vee s \leq-1 \wedge \widehat{s} \leq-1 \\
J<0, \widehat{J}<0, & 2 J+1 \leq s \leq-1 \wedge 2 \widehat{J}+1 \leq \widehat{s} \leq-1 \\
J \geq 0, \widehat{J}<0, & s \leq-1 \wedge \widehat{s} \leq-1 \vee s \geq 2 J+1 \wedge \widehat{s} \geq 2 \widehat{J}+1 \\
J<0, \widehat{J} \geq 0, & s \leq-1 \wedge \widehat{s} \leq-1 \vee s \geq 2 J+1 \wedge \widehat{s} \geq 2 \widehat{J}+1 .
\end{array}
$$

For these values of the parameters, poles of the normalizations $I_{m^{e}}^{\left(J^{e}\right)}$ occur. However, the full three point function has poles only for

$$
J \geq 0, \widehat{J}<0, \quad s \leq-1 \wedge \widehat{s} \leq 2 \widehat{J} \vee s \geq 2 J+1 \wedge \widehat{s} \geq 0
$$

and vice versa, while in the other cases, the singularities in $\left|I_{m^{e}}^{\left(J^{e}\right)}\right|^{2}$ are compensated by a vanishing factor from the residue calculation or directly from the coefficient $a_{n, \widehat{n}}^{\left(J^{e}\right)}$ in Eq. (12), and the product is ill-defined. In these cases, the correct result is obtained by taking a limit from continous $J^{e}: 2 \square$ this will be shown in the next paragraph. The parameter range Eq. (87) does not couple to the other regions and can thus be suppressed consistently.

\subsection{Reflection amplitude}

Let us start from the trivial observation that the weight formula for the chiral fields $V_{m}^{\left(J^{e}\right)}$ as well as the Liouville exponentials,

$$
\Delta_{J^{e}}=-J^{e}-\frac{h}{\pi} J^{e}\left(J^{e}+1\right)
$$

has two roots $J^{e}$ and $J_{r}^{e}=-\varpi_{0}-J^{e}$. For the corresponding ground states $\left|\varpi_{J^{e}}\right\rangle \equiv\left|\varpi_{0}+2 J^{e}\right\rangle$, the replacement

$$
J^{e} \rightarrow J_{r}^{e}=-\varpi_{0}-J^{e}
$$

\footnotetext{
${ }^{20}$ Recall that the $g_{J^{e}, x+m^{e}}^{x}$, understood as coupling constants for the chiral algebra, are not subject to the restriction Eq. (83) arising in our treatment of the nonchiral observables.
} 
amounts to $\varpi_{J^{e}} \rightarrow-\varpi_{J^{e}}$ and therefore the reflection invariance $\varpi_{f} \rightarrow$ $-\varpi_{f}, \varpi_{i} \rightarrow-\varpi_{i}$ of section 3.1 is generated by combining two such operations. As any primary conformal field is defined uniquely up to a zero mode dependent normalization by its conformal weight and its zero mode shift $m$ [24], we have the identity ${ }^{21}$

$$
V_{m}^{\left(J^{e}\right)}=V_{m}^{\left(J_{r}^{e}\right)}
$$

for the normalized operators $V_{m}^{\left(J^{e}\right)}$. On the other hand, the operation Eq. (89), which implies

$$
s^{e} \equiv s+\widehat{s} \frac{\pi}{h}=J^{e}+m^{e} \rightarrow J_{r}^{e}+m^{e}=s^{e}-2 J^{e}-\varpi_{0}
$$

always connects a point in Eq. (86) to a point where the residue calculation is well-defined, and vice versa. Thus, for example, the primaries appearing in the region $J \geq 0, \widehat{J} \geq 0, s \geq 2 J+1, \widehat{s} \geq 2 \widehat{J}+1$ contained in Eq. (86) are the same as those for $J<0, \widehat{J}<0, s \geq 0, \widehat{s} \geq 0$. But in the latter region, the analysis of ref. [8] tells us that locality fixes the $g \bar{g}$ coefficients up to the common similarity transformation $T(\varpi)$ already discussed, and a $J^{e}$ dependent c-number normalization constant. This means that the coefficients of the extension $s \geq 2 J+1, \widehat{s} \geq 2 \widehat{J}+1$ of the $J \geq 0, \widehat{J} \geq 0$ exponentials must agree up to a constant with those for $J<0, \widehat{J}<0, s \geq 0, \widehat{s} \geq 0$ which are known; similar remarks apply to the other cases. Invoking furthermore reflection invariance as in Eq. (63), which connects pairwise subcases with given $J, \widehat{J}$, one sees that there is only one $J^{e}$ - dependent constant that can be introduced. Thus we arrive at the conclusion that

$$
\exp \left[-J_{r}^{e} \alpha_{-} \Phi\right]=A_{R}\left(J^{e}\right) \exp \left[-J^{e} \alpha_{-} \Phi\right]
$$

for a suitable constant $A_{R}\left(J^{e}\right)$, which we will call reflection amplitude, in the same sense as in ref. [6]. One may now verify by tedious explicit calculations that Eq. (92) is fulfilled with the definition of the coefficients in the region Eq. (86) by limit from continous $J^{e}$ as proposed above. However, there is a much simpler argument allowing to determine the explicit value of $A_{R}\left(J^{e}\right)$ : Consider the joint reflection operation $J_{f}^{e} \rightarrow\left(J_{f}^{e}\right)_{r}, J_{i}^{e} \rightarrow\left(J_{i}^{e}\right)_{r}$, or

\footnotetext{
${ }^{21} \mathrm{~A}$ related reasoning was already used in ref. [7], where the symmetry of the braiding and fusion matrices under the reflection (89) was observed.
} 
$\varpi_{f} \rightarrow-\varpi_{f}, \varpi_{i} \rightarrow-\varpi_{i}$, which leaves the three point matrix element (17) invariant. Its only effect on the three point function therefore comes from the normalization factors $F_{i}, F_{f}$ in Eq. (18) and the cosmological constant dependence (cf. Eq. (69)), and we have

$$
\begin{gathered}
A_{R}\left(J_{f}^{e}\right) A_{R}\left(J_{i}^{e}\right)=\frac{\left\langle\exp \left[-\left(J_{f}\right)_{r} \alpha_{-} \Phi\right] \exp \left[-J_{r} \alpha_{-} \Phi\right] \exp \left[-\left(J_{i}\right)_{r} \alpha_{-} \Phi\right]\right\rangle}{\left\langle\exp \left[-J_{f} \alpha_{-} \Phi\right] \exp \left[-J_{-} \Phi\right] \exp \left[-J_{i} \alpha_{-} \Phi\right]\right\rangle}= \\
\left(\mu^{2} \frac{\pi \sin h}{8 h^{2}} \Gamma^{2}(1+h / \pi)\right)^{\varpi_{f}-\varpi_{i}} \frac{F_{f}\left(-\varpi_{f}\right) F_{i}\left(-\varpi_{i}\right)}{F_{f}\left(\varpi_{f}\right) F_{i}\left(\varpi_{i}\right)}
\end{gathered}
$$

from which we read off, using the permutation symmetry of the three point function and Eqs. (65), (24), that ${ }^{22}$

$$
\begin{gathered}
\frac{A_{R}\left(J^{e}\right)}{\left(\mu^{2} \frac{\pi \sin h}{8 h^{2}} \Gamma^{2}\left(1+\frac{h}{\pi}\right)\right)^{-\varpi_{0}-2 J^{e}}}=\frac{F_{f}\left(\varpi_{0}+2 J^{e}\right)}{F_{f}\left(-\varpi_{0}-2 J^{e}\right)}=\frac{F_{i}\left(-\varpi_{0}-2 J^{e}\right)}{F_{i}\left(\varpi_{0}+2 J^{e}\right)}= \\
-\epsilon_{J^{e}} \frac{\Gamma\left(2+\frac{\pi}{h}+2 J^{e}\right) \Gamma\left(2+\frac{h}{\pi}+2 \widehat{J}^{e}\right)}{\Gamma\left(-2 J^{e}-\frac{\pi}{h}\right) \Gamma\left(-2 \widehat{J}^{e}-\frac{h}{\pi}\right)}
\end{gathered}
$$

The factor $\epsilon_{J}$ is equal to 1 when $J \geq 0, \widehat{J}<0$ or vice versa, where the factors $\frac{1}{2}$ arising from the residue contributions cancel; for $J, \widehat{J} \geq 0$ or $J, \widehat{J}<0$ it takes the values $\frac{1}{4}$ and 4, respectively. Eq. (94) agrees with the reflection amplitude proposed in ref. [6] [3]; the comparison with the DOZZ conjecture will be discussed in more detail in section 5. The equality of the reflection amplitude to the ratio of the normalization factors $F_{f}$ or $F_{i}$ appearing in Eq. (18) has an important consequence: It implies that the matrix element (17), where $F_{f, i}$ are divided out, is invariant not only under the joint reflection $\varpi_{f} \rightarrow-\varpi_{f}, \varpi_{i} \rightarrow-\varpi_{i}$ considered in section 3.1, but under $\varpi_{f} \rightarrow-\varpi_{f}$ and $\varpi_{i} \rightarrow-\varpi_{i}$ separately. Consideration of the case $\varpi_{f}=0$ or $\varpi_{i}=0$ shows that we have symmetric and not antisymmetric behaviour, and thus the sign choice in Eq. (94) is correct. This leads us to identify the ground states with opposite values of the zero mode:

$$
|\varpi\rangle \cong|-\varpi\rangle
$$

\footnotetext{
${ }^{22}$ up to a sign; we will show below that the choice in Eq. (94) is the right one.

${ }^{23}$ except for the factor $\epsilon_{J^{e}}$, cf. section 5 .
} 
Such an identification is of course expected since the map from the Liouville field to the free field is two to one, and the ground states $|\varpi\rangle$ and $|-\varpi\rangle$ have the same conformal weight. However, as we have seen, its validity requires rather nontrivial properties of the Liouville exponentials and therefore could not yet be established in previous analyses. Our argument is based entirely on the three point function, which however should be sufficient in a conformal theory $[30]^{24}$

At this point, a general remark about the extensions Eq. (86) is in order. While Eq. (92) is valid only when the latter are taken into account, we know that, for instance, the unextended exponentials with $J \geq 0, \widehat{J} \geq 0$ are perfectly consistent with locality as they are degenerate fields. It is thus the full realization of reflection invariance which requires the extensions. A very similar remark applies to the (two-screened) exponentials with $\widehat{J}=0$ (or

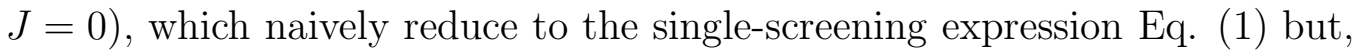
according to Eq. (86), actually retain summations with nonzero $\widehat{n}$ (or $n$ ).

Summarizing, we conclude that the expansion Eq. (14) should be extended to the regions Eq. (86) without Eq. (87) by taking limits from continous $J^{e}$ or making use of Eq. (92), and this defines the summation ranges in Eq. (80). According to the remark above Eq. (85), we have $\delta_{J}=1$ for $J \geq 0, \widehat{J} \geq 0, \delta_{J^{e}}=\frac{1}{4}$ for $J<0, \widehat{J}<0$, and $\delta_{J^{e}}=\frac{1}{2}$ otherwise.

We close this section with a remark on the question of the $S L(2)$ invariant vacuum mentioned in the footnote on Eq. (18). The prescription (18) appears to break reflection invariance by its preference of the $\vartheta_{1}$ representation with respect to the vacuum $\left|\varpi_{0}\right\rangle$. On the other hand, the above extensions of the Liouville sums of Eq. (12) tell us that even in the case $\widehat{J}=0$, the shifts $m^{e}=-J^{e}$ and $m^{e}=-J_{r}^{e}$ always appear together in any given exponential. The corresponding operators have the same $z \rightarrow 0$ behaviour on the vacuum $\left|\varpi_{0}\right\rangle$, and thus actually two highest weight states $\left|\varpi_{f}\right\rangle,\left|-\varpi_{f}\right\rangle$ and $\left|\varpi_{i}\right\rangle,\left|-\varpi_{i}\right\rangle$ are created instead of just one as supposed in Eq. (18). It is easy to check that their coefficients are equal, so that no breaking of reflection invariance

\footnotetext{
${ }^{24}$ Actually there is the subtle matter of factorization for the Liouville four point function [18] [9]; however, here by definition there is no such problem because we factorize over elliptic states - cf. section 6 .

${ }^{25}$ Actually, it is slightly more appropriate to speak of $n, \widehat{n}$ rather than $s, \widehat{s}$ in Eq. (86) as these values are not related to residue calculations but should be included in the initial sum Eq. (14); however, thinking in terms of effective representations of the form Eq. (80), the difference is of little relevance.
} 
actually occurs! As the two highest weight states can be identified according to Eq. (95), the net effect of the "forgotten" contribution for the three point function is just a global factor of 2 independent of all spins. Moreover, one can verify that using the reflected left and right vacua $\left|\mp \varpi_{0}\right\rangle$ instead of $\left| \pm \varpi_{0}\right\rangle$ leads to the same result, as a consequence of the reflection symmetry of the exponentials. This can be taken as a hint that, although there does not truly exist an $S L(2)$ - invariant vacuum in the Hilbert space because on each of the candidate vacua $\left| \pm \varpi_{0}\right\rangle$ one of the free fields becomes singular [21], the physical observables may be shielded from this problem by reflection invariance and behave as if both vacua $\left| \pm \varpi_{0}\right\rangle$ were truly $S L(2)$ - invariant 20. We hope to discuss this question in greater detail elsewhere.

\section{Comparison with DOZZ}

We are now in a position to compare our results with the DOZZ conjecture. Our discussion will be brief since a detailed comparison between the coupling constants $g_{J, x+m}^{x} \bar{g}_{J, x+m}^{x}$ describing the three point function and the DOZZ result has been carried out in ref. [15]. The DOZZ result in the form of ref. [6] reads

$$
\begin{aligned}
& \left\langle\exp \left[-J_{f} \alpha_{-} \Phi\right] \exp \left[-J \alpha_{-} \Phi\right] \exp \left[-J_{i} \alpha_{-} \Phi\right]\right\rangle_{\mu^{2}}=\left[\pi \mu \gamma\left(b^{2}\right) b^{2-2 b^{2}}\right]^{\left(Q-\sum_{k=1}^{3} \alpha_{k}\right) / b} \\
& \times \frac{\Upsilon_{0} \Upsilon\left(2 \alpha_{1}\right) \Upsilon\left(2 \alpha_{2}\right) \Upsilon\left(2 \alpha_{3}\right)}{\Upsilon\left(\alpha_{1}+\alpha_{2}+\alpha_{3}-Q\right) \Upsilon\left(\alpha_{1}+\alpha_{2}-\alpha_{3}\right) \Upsilon\left(\alpha_{2}+\alpha_{3}-\alpha_{1}\right) \Upsilon\left(\alpha_{3}+\alpha_{1}-\alpha_{2}\right)}
\end{aligned}
$$

Here, we have

$$
b=\sqrt{\frac{h}{\pi}}, \alpha_{k}=-b J_{k}, Q=b+\frac{1}{b}=b \varpi_{0}
$$

The symbol $\Upsilon$ denotes a new special function for which integral representations were given in [28] [6]. It fulfills the functional relations

$$
\begin{gathered}
\Upsilon(x+b)=\gamma(b x) b^{1-2 b x} \Upsilon(x) \\
\Upsilon(x+1 / b)=\gamma(x / b) b^{2 x / b-1} \Upsilon(x)
\end{gathered}
$$

\footnotetext{
${ }^{26}$ This would fit in well with the ideas in [32] where it is proposed to formulate the theory on states corresponding to Kac's zeroes in terms of Verma rather than Fock modules.
} 
with $\gamma(x):=\frac{\Gamma(x)}{\Gamma(1-x)}$, as well as

$$
\Upsilon(x)=\Upsilon(Q-x), \quad \Upsilon(Q / 2)=1 .
$$

Finally, $\Upsilon_{0}=\left.\frac{d \Upsilon(x)}{d x}\right|_{x=0}$. The $\Upsilon$ function, and thus the formula Eq. (96), is explicitly symmetric in $\alpha_{+}$and $\alpha_{-}$, or $h$ and $\widehat{h}=\frac{\pi^{2}}{h}$, and thus supports our interpretation of the symmetric expansion Eq. (12) as a different representation of the Liouville exponential rather than a new observable. The recursion relations Eq. (98) were shown in ref. [16] to follow from crossing symmetry, as mentioned at the end of section 3. The pole and zero structure of the expression (96) is determined in terms of the zeroes of the function $\Upsilon(x)$, which lie at

$$
x=-b\left(n+\widehat{n} \frac{\pi}{h}\right)
$$

with $n, \widehat{n}$ both nonnegative or both negative. Let us consider Eq. (96) as a function of the screening charge $s^{e}=\varpi_{0}+\sum_{k} J_{k}^{e}=-\frac{1}{b}\left(\sum_{k} \alpha_{k}-Q\right)$. For greater simplicity of comparison, we consider generic continous values of $J_{f}^{e}, J_{i}^{e}$ (subject to condition (11)) though we can be sure of the validity of relations (18) only if $J_{f}, J_{i}$ also fulfill Eq. (33).므 Then the position of poles and zeroes in Eq. (96) as a function of $s^{e}$ does not depend on $J_{f}, J_{i}$; as mentioned in the introduction, Eq. (96) exhibits poles in $s^{e}$ at

$$
s \geq 0, \widehat{s} \geq 0 \vee s<0, \widehat{s}<0 \vee s \leq 2 J, \widehat{s} \leq 2 \widehat{J} \vee s \geq 2 J+1, \widehat{s} \geq 2 \widehat{J}+1
$$

due to the factors $\Upsilon\left(\sum_{k} \alpha_{k}-Q\right) \equiv \Upsilon(-b s)$ and $\Upsilon\left(\alpha_{3}+\alpha_{1}-\alpha_{2}\right) \equiv \Upsilon(-b(2 J-$ $s)$ ) in the denominator, which however may be (partially) compensated by a vanishing factor $\Upsilon\left(2 \alpha_{2}\right)$ in the numerator when $J \geq 0, \widehat{J} \geq 0$ or $J<$ $0, \widehat{J}<0$. In these cases, according to ref. 15 one should define the ratios $\Upsilon\left(2 \alpha_{2}\right) / \Upsilon\left(\sum_{k} \alpha_{k}-Q\right)$ and $\Upsilon\left(2 \alpha_{2}\right) / \Upsilon\left(\alpha_{3}+\alpha_{1}-\alpha_{2}\right)$ for $s \geq 0, \widehat{s} \geq 0$ or $s<0, \widehat{s}<0$ and $s \leq 2 J, \widehat{s} \leq 2 \widehat{J}$ or $s \geq 2 J+1, \widehat{s} \geq 2 \widehat{J}+1$, respectively, by formal cancellation of the singular factors $\Upsilon(0)$ remaining in numerator and denominator after using the recurrence relations. Now our result Eq. (66), on the other hand, is finite at all of the points Eq. (101). The difference is due to the fact that, as remarked below Eq. (41), we have been working with a discrete zero mode scalar product. We should thus compare Eq. (69) with the residues of the DOZZ three point function at these poles, which fulfill the

\footnotetext{
${ }^{27}$ Recall that our result for the matrix element (17) is truly valid for continous $\varpi_{f}, \varpi_{i}$.
} 
same crossing symmetry recursion relations as for the nonsingular points. In doing this, we employed a very useful observation made in ref. [15]; there it is shown that expression (96) is the interpolating function extending the coupling constants in Eq. (60) to continous spins. More precisely, the DOZZ three point function is a meromorphic function whose residues at the poles in $s^{e}$ agree with the values of the coupling constants $g \bar{g}$. We thus find that at the above points, Eqs. (66) and (96) agree up to an overall constant independent of all the spins, except for the factors $\frac{1}{2}, \frac{1}{4}$ arising from the Cauchy principal value prescription for the residue contributions. This seems to imply that for the continous spectrum theory, the $i \epsilon$ prescription for the treatment of the $z$ - poles Eq. (51) is favoured, which leads to a unit normalization of the exponentials with $J<0$ and/or $\widehat{J}<0$. Consequently, the leading order operator product, which was used to determine the latter, should also differ by a factor of two resp. four from the one in the continous theory, in the relevant cases. This may seem surprising, but we must keep in mind that the system of intermediate states defining the operator product is rather different in the two theories. In fact, as suggested in [18 [9] and supported by the analysis of [6], in the continous theory the system of intermediate states should actually be parametrized by continous imaginary values of $\varpi$, the socalled hyperbolic states (corresponding to complex spins $J=-\varpi_{0} / 2+i p$ ), while in our analysis we have discrete real values of $\varpi$. The reason why the discrepancy consists just in a constant factor depending only on the sign of $J, \widehat{J}$ is that the crossing symmetry relations obtained from the null vector decoupling equations are the same for the continous and the discrete theory, and thus allow for a change of normalization in the latter only when traversing the points $J=0$ resp. $\widehat{J}=0$ where they become ill-defined.

It remains to analyze the points

$$
\begin{array}{ll}
J \geq 0, \widehat{J} \geq 0, & 0 \leq s \leq 2 J \wedge(\widehat{s} \geq 2 \widehat{J}+1 \vee \widehat{s} \leq-1) \text { and } h \leftrightarrow \widehat{h} \\
J<0, \widehat{J}<0, & 2 J+1 \leq s \leq-1 \wedge(\widehat{s} \leq 2 \widehat{J} \vee \widehat{s} \geq 0) \text { and } h \leftrightarrow \widehat{h} \\
J \geq 0, \widehat{J}<0, & s \geq 2 J+1 \wedge \widehat{s} \leq 2 \widehat{J} \vee s \leq-1 \wedge \widehat{s} \geq 0 \\
& \vee 0 \leq s \leq 2 J \wedge 2 \widehat{J}+1 \leq \widehat{s} \leq-1 \\
\text { and } h \leftrightarrow \widehat{h} &
\end{array}
$$

where the DOZZ formula predicts finite nonvanishing values. The cases with $J \geq 0, \widehat{J} \geq 0$ and $J<0, \widehat{J}<0$ are connected by the reflection operation, and the same applies to the cases with $J \geq 0, \widehat{J}<0$ and $J<0, \widehat{J} \geq 0$. Looking 
at Eqs. (57), (54) or their "hatted" counterparts, we see that in these cases one of the residue sums $R_{0}+R_{1}, \widehat{R}_{0}+\widehat{R}_{1}$ vanishes while the normalizations $I_{m^{e}}^{\left(J^{e}\right)}(\varpi)$ are finite, so that our three point function vanishes. This is perfectly consistent with the structure of the chiral coupling constants $g_{J, x+m}^{x}, \bar{g}_{J, x+m}^{x}$ in this region [27]. Thus, the discrete theory constructed here lives only on the poles of the DOZZ three point function. The above Liouville exponentials should be viewed as the direct generalization of those for $2 J$ positive integer considered in the older works [11], where the spectrum was also assumed to be discrete. However, the resulting conformal field theory is obviously a truncation of the full Liouville theory which has a continous spectrum. To derive the nonvanishing finite values of the DOZZ three point function at the points Eq. (102) from our approach, we thus would need a regularization prescription to balance the infinite zero mode volume against the vanishing of the residue sum; this is the well-known problem of coupling constant renormalization, which appears in a different guise also in the Goulian/Li approach [1] 2] 3]. At present, we cannot derive the proper regularization procedure from first principles. This is because any systematic regularization of the zero mode integration seems to require going away at least infinitesimally from the integer screening points Eq. (11). We would then need expansions of the Liouville exponentials with a general continous monodromy, which, as explained in section 4 , is a nontrivial problem. However, it is of course possible to formally reproduce the DOZZ three point function at the above points as well in the operator approach. Indeed, the main technical ingredients of the latter are the algebra of the chiral operators $V_{m}^{(J)}$ and the locality condition. We could therefore start with an ansatz of the form Eq. (80) and try to determine the coefficients such that locality is fulfilled in the region Eq. (102). The solution is easy to obtain by a formal renormalization of the coefficients $g_{J, x+m}^{x} \bar{g}_{J, x+m}^{x}$ above. Indeed, the expressions $R_{0}, R_{1}$ (and their hatted counterparts) where the zeroes occur, are given by finite products with one vanishing factor. Taking out this factor produces a solution of the crossing symmetry conditions as written in ref. [16]2 , and one obtains agreement with DOZZ again. From this point of view, the problem becomes a matter of imposing boundary conditions on the solutions of the locality, or crossing symmetry, conditions; in other words, we need to decide on which

\footnotetext{
${ }^{28}$ This is not very surprising as the crossing symmetry conditions involve only ratios of coefficients.
} 
set of points the solution should be vanishing resp. finite. We would like to comment here on an older result by Gervais for the three point function of minimal matter coupled to gravity [7]. There dressing operators were introduced which are local and have the correct conformal dimension, but looked very different from the Liouville exponentials that were expected to play this role, and so their meaning was not obvious. In particular, they are not singlets under the quantum group ${ }^{29}$. From the present analysis, it is now clear that they should be viewed as effective representations of the Liouville exponentials for the infinite volume case, at integer "off-shell" values of the spins away from the poles of the DOZZ three point function; in other words, they correspond to a restriction of Eq. (80) with renormalized coupling constants to a subset of Eq. (102). Explicitly, the local operators of [7] can be written as the fusion of a standard exponential of the form Eq. (11) with screening charge $\alpha_{+}$and $2 \widehat{J}$ positive integer, and a new local field involving $\alpha_{-}$, or vice versa. The latter can be written in the form

$$
\exp \left[-J \alpha_{-} \breve{\Phi}\right]=C_{J} \sum_{n=2 J+1}^{-1} \frac{T(\varpi)}{T(\varpi+2 m)} \breve{g}_{J, x+m}^{x} \breve{\breve{g}}_{J, x+m}^{x} V_{m}^{(J)} \bar{V}_{m}^{(J)}
$$

with $2 J$ a negative integer, and $n=J+m$. We see therefore that we are in the situation $J<0, \widehat{J} \geq 0,2 J+1 \leq s \leq-1 \wedge 0 \leq \widehat{s} \leq 2 \widehat{J}$ contained in Eq. (102).

Moreover, one can show easily that the coefficients $\breve{g}_{J, x+m}^{x} \breve{\breve{g}}_{J, x+m}^{x}$ as given in ref. [7] agree precisely with the renormalized coupling constants defined above. Thus we can identify

$$
\exp \left[-J \alpha_{-} \breve{\Phi}\right] \equiv \exp \left[-J \alpha_{-} \Phi\right]_{\text {ren }}
$$

as claimed, and similarly for the fusion with $e^{-\widehat{J} \alpha_{+} \Phi}$. We note that the renormalized exponentials are to be treated using the finite volume zero mode scalar product, as the infinite volume already went into the renormalization of the coupling constants.

\footnotetext{
${ }^{29}$ Already classically, the invariance under $A \rightarrow A+c, B \rightarrow B+c$ (cf. Eq. (20) ) is broken.
} 


\section{Conclusions/Outlook}

Two main lessons are to be learnt from the above analysis: First, there is a consistent truncation of Liouville theory that involves integer powers of screenings, or highest/lowest weight representations of $U_{q}(s l(2))$, only. It is for this truncated theory that the expansions of ref. [8] can immediately be used; however, their evaluation turned out to be rather delicate and required a nonperturbative definition of the infinite chiral decompositions representing the observables. The main principles ensuring that this problem has a welldefined solution were seen to be locality and reflection invariance; the latter leads to a symmetric presence of highest and lowest weight representations, or both of the two equivalent free fields. The general message from this is that while Coulomb gas type representations similar to the ones familiar from rational conformal field theories continue to work for the chiral operator algebra in the present irrational theory, the appearance of an infinite number of primaries in the nonchiral observables leads to new nonperturbative effects which break charge conservation. Within the family of discrete spectrum truncations of Liouville theory discussed here, this breaking is not arbitrary but can be parametrized in terms of negative screening powers. Remarkably, the Gervais-Neveu quantization scheme provides us from the beginning with the appropriate technical machinery to describe the resulting generalized Coulomb gas picture and gives an explicit operator representation of positive and negative screenings on the same footing; this should, in particular, make further progress possible for $N \geq 4$ point functions by exploiting the conformal and group-theoretic properties of this representation.

Second, in order to obtain the three point function in the most general situation, according to the principle of monodromy invariance one has to study the algebra of arbitrary powers of screenings, or general discrete representations of $U_{q}(s l(2))$. Technically, what is required for the general case is an extension of the $q-6 j$ symbols to noninteger screening numbers in such a way that the polynomial equations of Moore and Seiberg [33] remain valid, together with a Coulomb gas picture for arbitrary screening powers along the lines of [17]. The final result should be a decomposition formula for the Liouville exponentials in terms of arbitrary representations. This would be very important in particular in order to resolve the problem of a rigorous treatment of the zero mode integration in the continous spectrum case, and to address the issue of factorization. Indeed, from general arguments [9] [18] 
one expects the four point function to factorize over hyperbolic states - corresponding to imaginary $\varpi-$ and this is supported by the analysis of [6]. On the other hand, in the present analysis we have factorized over elliptic states ( $\varpi$ real) by means of the coherent state projector. It may well be that this is truly consistent only in the truncated theory; in any case, the meaning of the poles contributing to our residue integral needs to be better understood. Finally, the issue of the Seiberg bound still awaits its resolution; neither in the DOZZ analysis nor in the present one has it been seen to play any significant role so far. However, it may surface once we analyze the operator product of the Liouville exponentials - cf. the remark in section 3.4 - which is expected to have a rather nontrivial structure in the general case. We leave these interesting questions to further studies.

Acknowledgements It is a pleasure to thank Jean-Loup Gervais for the fruitful collaboration on the earlier work and useful discussions and criticisms on the present one. I am also grateful for helpful discussions to L. AlvarezGaume and S. Shatashvili.

\section{Appendix}

Here we give the explicit formulae for the normalizations $I_{m}^{(J)}(\varpi)$ which were derived in ref. [8]. In the case $m=n-J, n=0,1,2, \ldots$, we have

$$
\begin{gathered}
I_{m}^{(J)}(\varpi)=\left(2 \pi \Gamma\left(1+\frac{h}{\pi}\right)\right)^{n} e^{i h n(\varpi-J+m)} \\
\times \prod_{l=1}^{n} \frac{\Gamma(1+(2 J-l+1) h / \pi)}{\Gamma(1+l h / \pi) \Gamma(1-(\varpi+2 m-l) h / \pi) \Gamma(1+(\varpi+l) h / \pi)}
\end{gathered}
$$

for any continous value of $J$. For $n<0$, Eq. (105) is still valid if we define as usual $\prod_{l=1}^{-|n|} f(l):=1 / \prod_{l=1}^{|n|} f(l-|n|)$. If both screening charges are present, so that $m=-J+n+\widehat{n} \pi / h$, one obtains

$$
\begin{gathered}
I_{m \widehat{m}}^{(J \widehat{J})}(\varpi)=I_{m^{\circ}}^{\left(J^{e}\right)}\left(\varpi+2 \widehat{n} \frac{\pi}{h}\right) \widehat{I}_{\widehat{m}^{\circ}}^{\left.\widehat{J}^{e}\right)}\left(\widehat{\varpi}+2 n \frac{h}{\pi}\right)\left(i \frac{\pi}{h}\right)^{2 n \widehat{n}} \times \\
\prod_{l, \hat{l}=1}^{n, \widehat{n}}\left\{\left(l+\hat{l} \frac{\pi}{h}\right)\left(\varpi+2 m+2 \widehat{m} \frac{\pi}{h}-l-\hat{l} \frac{\pi}{h}\right)\left(\varpi+l+\hat{l} \frac{\pi}{h}\right)\left(2 J^{e}-(l-1)-(\hat{l}-1) \frac{\pi}{h}\right)\right\}^{-1}
\end{gathered}
$$




$$
\times \prod_{\hat{l}=1}^{2 \widehat{n}} \prod_{l=1}^{n}\left(\hat{l}+(\varpi+l) \frac{h}{\pi}\right) \prod_{l=1}^{2 n} \prod_{\hat{l}=1}^{\widehat{n}}\left(l+(\widehat{\varpi}+\hat{l}) \frac{\pi}{h}\right)
$$

for nonnegative $n, \widehat{n}$. The continuation to negative $n$ or $\widehat{n}$ works in the same way as for Eq. (105).

\section{References}

[1] M. Goulian, M. Li, Phys. Rev. Lett. 66 (1991) 2051.

[2] Vl.S. Dotsenko, Mod. Phys. Lett. 6 (1991) 3601.

[3] P. Di Francesco, D. Kutasov, Nucl. Phys. B375 (1992) 119.

[4] M. Bershadsky, I. Klebanov, Phys. Rev. Lett. 65 (1990) 3088.

[5] H. Dorn, H.-J. Otto, Nucl. Phys. B429 (1994) 375.

[6] A.B. Zamolodchikov, Al.B. Zamolodchikov, hep-th/9506136.

[7] J.-L. Gervais, Nucl. Phys. B391 (1993) 287 and references therein.

[8] J.-L. Gervais, J. Schnittger, Nucl. Phys. B431 (1994) 273.

[9] N. Seiberg, Progr. Theor. Phys. Suppl. 102 (1990) 319.

[10] P. Ginsparg, G. Moore, Lectures given at the 1992 TASI summer school, hep-th/9304011.

[11] J.-L. Gervais, A. Neveu, Nucl. Phys. B224 (1983) 329; Nucl. Phys. B238 (1984) 125; Nucl. Phys. B238 (1984) 396.

[12] J.-L. Gervais, J.-F. Roussel, Nucl. Phys. B426 (1994) 140.

[13] J.-L. Gervais, J. Schnittger, Phys. Lett. B315 (1993) 258.

[14] E. Cremmer, J.-L. Gervais, J.-F. Roussel, Nucl. Phys. B413 (1994) 244.

[15] J.-L. Gervais, "The Liouville Coupling Constants", to be published. 
[16] J. Teschner, "On the Liouville Three-Point Function", hep-th/9507109.

[17] J.L. Petersen, J. Rasmussen, M. Yu, "Conformal Blocks for Admissible Representations in SL(2) Current Algebra", hep-th/9504127.

[18] J. Polchinski, "Remarks on Liouville field theory", in "Strings 90", eds. R. Arnowitt et al., World Scientific 1991.

[19] J.-L. Gervais, J. Schnittger, Nucl. Phys. B413 (1994) 277.

[20] J.-L. Gervais, A. Neveu, Nucl. Phys. B224 (1983) 329.

[21] D. Luest, J. Schnittger, Int. J. Mod. Phys. A6 (1991) 3625.

[22] A. Bilal, J.-L. Gervais, Nucl. Phys. B318 (1989) 579.

[23] A. Anderson, B.E.W. Nilsson, C.N. Pope and K.S. Stelle, Nucl. Phys. B430 (1994) 107.

[24] J.-L. Gervais, A. Neveu, Nucl. Phys. B238 (1984) 125.

[25] N.Ja. Vilenkin, A.U. Klimyk, "Representation of Lie Groups and special Functions" vol.3, Kluwer Academic Publishers 1992.

[26] H. Exton, "Q-hypergeometric Functions and Applications", E. Horwood Ltd. 1983.

[27] J. Schnittger, unpublished calculations.

[28] H.J. Otto, G. Weigt, Z. Phys. C31 (1986) 219.

[29] F. David, Mod. Phys. Lett. A3 (1988) 1651; J. Distler, H. Kawai, Nucl. Phys. B321 (1989) 509.

[30] A.A. Belavin, A.M. Polyakov, A.B. Zamolodchikov, Nucl. Phys. B241 (1984) 332.

[31] P. Ginsparg, G. Moore, 1992 TASI lectures, hep-th/9304011.

[32] J. Teschner, Ph.D. thesis, preprint DESY 95-118.

[33] G. Moore, N. Seiberg, Comm. Math. Phys. 123 (1989) 77. 